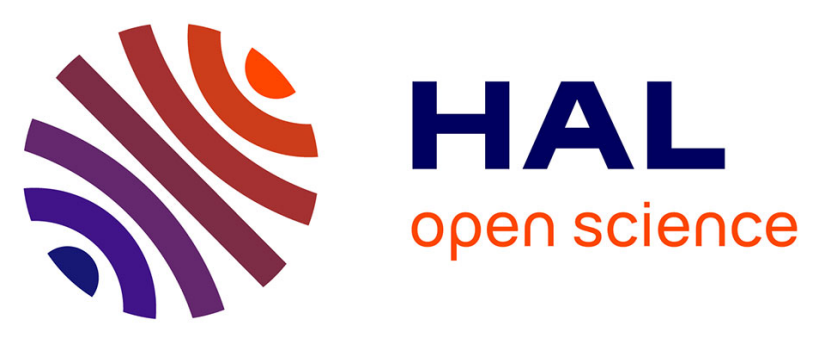

\title{
Different Clinical Contexts of Use of Blood Neurofilament Light Chain Protein in the Spectrum of Neurodegenerative Diseases
}

Giovanni Palermo, Sonia Mazzucchi, Alessandra Della Vecchia, Gabriele Siciliano, Ubaldo Bonuccelli, Carole Azuar, Roberto Ceravolo, Simone Lista, Harald Hampel, Filippo Baldacci

\section{To cite this version:}

Giovanni Palermo, Sonia Mazzucchi, Alessandra Della Vecchia, Gabriele Siciliano, Ubaldo Bonuccelli, et al.. Different Clinical Contexts of Use of Blood Neurofilament Light Chain Protein in the Spectrum of Neurodegenerative Diseases. Molecular Neurobiology, 2020, 57 (11), pp.4667-4691. 10.1007/s12035020-02035-9 . hal-03385896

\section{HAL Id: hal-03385896 \\ https: / hal.sorbonne-universite.fr/hal-03385896}

Submitted on 19 Oct 2021

HAL is a multi-disciplinary open access archive for the deposit and dissemination of scientific research documents, whether they are published or not. The documents may come from teaching and research institutions in France or abroad, or from public or private research centers.
L'archive ouverte pluridisciplinaire HAL, est destinée au dépôt et à la diffusion de documents scientifiques de niveau recherche, publiés ou non, émanant des établissements d'enseignement et de recherche français ou étrangers, des laboratoires publics ou privés. 


\section{Dear Author}

Here are the proofs of your article.

- You can submit your corrections online or by fax.

- For online submission please insert your corrections in the online correction form. Always indicate the line number to which the correction refers.

- Please return your proof together with the permission to publish confirmation.

- For fax submission, please ensure that your corrections are clearly legible. Use a fine black pen and write the correction in the margin, not too close to the edge of the page.

- Remember to note the journal title, article number, and your name when sending your response via e-mail, fax or regular mail.

- Check the metadata sheet to make sure that the header information, especially author names and the corresponding affiliations are correctly shown.

- Check the questions that may have arisen during copy editing and insert your answers/corrections.

- Check that the text is complete and that all figures, tables and their legends are included. Also check the accuracy of special characters, equations, and electronic supplementary material if applicable. If necessary refer to the Edited manuscript.

- The publication of inaccurate data such as dosages and units can have serious consequences. Please take particular care that all such details are correct.

- Please do not make changes that involve only matters of style. We have generally introduced forms that follow the journal's style.

Substantial changes in content, e.g., new results, corrected values, title and authorship are not allowed without the approval of the responsible editor. In such a case, please contact the Editorial Office and return his/her consent together with the proof.

- If we do not receive your corrections within $\mathbf{4 8}$ hours, we will send you a reminder.

\section{Please note}

Your article will be published Online First approximately one week after receipt of your corrected proofs. This is the official first publication citable with the DOI. Further changes are, therefore, not possible.

After online publication, subscribers (personal/institutional) to this journal will have access to the complete article via the DOI using the URL:

http://dx.doi.org/10.1007/s12035-020-02035-9

If you would like to know when your article has been published online, take advantage of our free alert service. For registration and further information, go to: http://www.springerlink.com.

Due to the electronic nature of the procedure, the manuscript and the original figures will only be returned to you on special request. When you return your corrections, please inform us, if you would like to have these documents returned.

The printed version will follow in a forthcoming issue. 
32
Family Name Vecchia

Particle

Given Name

Suffix

Organization University of Pisa

Division

Address e-mail

Family Name

Particle

Given Name

Suffix

Author

Organization

Division

Address

e-mail

Family Name

Particle

Given Name

Suffix

Author

Organization

Division

Address

e-mail

Family Name

Particle

Given Name

Suffix

Organization

Division

Address

Organization

Division

Address

e-mail

Family

Family Name

Particle

\section{Ceravolo}

Azuar

\section{Alessandra Della}

Department of Clinical and Experimental Medicine

Pisa, Italy

\section{Siciliano}

\section{Gabriele}

University of Pisa

Department of Clinical and Experimental Medicine

Pisa, Italy

\section{Bonuccelli}

\section{Ubaldo}

University of Pisa

Department of Clinical and Experimental Medicine

Pisa, Italy

\section{Carole}

\section{AP-HP - Hôpital Pitié-Salpêtrière}

Centre National de Référence des Démences Rares ou Précoces, IM2A, Département de Neurologie

Paris, France

Institut du Cerveau et de la Moelle épinière (ICM)

FrontLab

Paris, France 
69

70

71

72

73

74

75

76

77

78

79

80

81

82

83

84

85

86

87

88

89

90

91

92

93

94

95

96

97

98

99

100
Given Name

Suffix

Organization

Division

Address

e-mail

Family Name

Particle

Given Name

Suffix

Organization

Division

Address

Author

Author

(n)

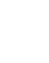

85

86

87

8

e-mail

-

Particle

1

2

\begin{tabular}{lll} 
& Particle & \\
Given Name & Harald \\
Suffix & \\
Organization & Institut du Cerveau et de la Moelle épinière (ICM) \\
Division & FrontLab \\
Address & Paris, France \\
\hline & e-mail & \\
\hline Schedule & Received & 10 May 2020 \\
& Revised & \\
\hline
\end{tabular}

4

5

Abstract

\section{Roberto}

University of Pisa

Department of Clinical and Experimental Medicine

Pisa, Italy

Lista

Simone

Sorbonne University

GRC $n^{\circ}$ 21, Alzheimer Precision Medicine (APM), AP-HP, Pitié-Salpêtrière Hospital

Boulevard de l'hôpital, Paris, France

Brain \& Spine Institute (ICM)

INSERM U 1127, CNRS UMR 7225

Boulevard de l'hôpital, Paris, France

Pitié-Salpêtrière Hospital

Department of Neurology, Institute of Memory and Alzheimer's Disease (IM2A)

Paris, France

\section{Hampel}

One of the most pressing challenges in the clinical research of neurodegenerative diseases (NDDs) is the validation and standardization of pathophysiological biomarkers for different contexts of use (CoUs), such as early detection, diagnosis, prognosis, and prediction of treatment response. Neurofilament light chain (NFL) concentration is a particularly promising candidate, an indicator of axonal degeneration, which can be analyzed in peripheral blood with advanced ultrasensitive methods. Serum/plasma NFL 
concentration is closely correlated with cerebrospinal fluid NFL and directly reflects neurodegeneration within the central nervous system. Here, we provide an update on the feasible CoU of blood NFL in NDDs and translate recent findings to potentially valuable clinical practice applications. As NFL is not a disease-specific biomarker, however, blood NFL is an easily accessible biomarker with promising different clinical applications for several NDDs: (1) early detection and diagnosis (i.e., amyotrophic lateral sclerosis, CreutzfeldtJakob disease, atypical parkinsonisms, sporadic late-onset ataxias), (2) prognosis (Huntington's disease and Parkinson's disease), and (3) prediction of time to symptom onset (presymptomatic mutation carriers in genetic Alzheimer's disease and spinocerebellar ataxia type 3).

101 Keywords separated Alzheimer's disease - Amyotrophic lateral sclerosis - Biomarkers by ' - ' Creutzfeldt-Jakob disease - NFL - Parkinsonian syndromes

102 Foot note Springer Nature remains neutral with regard to jurisdictional claims in information published maps and institutional affiliations. 


\title{
Different Clinical Contexts of Use of Blood Neurofilament Light Chain Protein in the Spectrum of Neurodegenerative Diseases
}

\author{
Giovanni Palermo ${ }^{1}$. Sonia Mazzucchi ${ }^{1}$ • Alessandra Della Vecchia ${ }^{1} \cdot$ Gabriele Siciliano $^{1}$ • Ubaldo Bonuccelli ${ }^{1}$. \\ Carole Azuar $^{2,3} \cdot$ Roberto Ceravolo $^{1}$. Simone Lista ${ }^{4,5,6} \cdot$ Harald Hampel $^{3} \cdot$ Filippo Baldacci $^{1,3}$
}

Received: 10 May 2020 / Accepted: 22 July 2020

(C) Springer Science+Business Media, LLC, part of Springer Nature 2020

\begin{abstract}
One of the most pressing challenges in the clinical research of neurodegenerative diseases (NDDs) is the validation and standardization of pathophysiological biomarkers for different contexts of use (CoUs), such as early detection, diagnosis, prognosis, and prediction of treatment response. Neurofilament light chain (NFL) concentration is a particularly promising candidate, an indicator of axonal degeneration, which can be analyzed in peripheral blood with advanced ultrasensitive methods. Serum/plasma NFL concentration is closely correlated with cerebrospinal fluid NFL and directly reflects neurodegeneration within the central nervous system. Here, we provide an update on the feasible CoU of blood NFL in NDDs and translate recent findings to potentially valuable clinical practice applications. As NFL is not a disease-specific biomarker, however, blood NFL is an easily accessible biomarker with promising different clinical applications for several NDDs: (1) early detection and diagnosis (i.e., amyotrophic lateral sclerosis, Creutzfeldt-Jakob disease, atypical parkinsonisms, sporadic late-onset ataxias), (2) prognosis (Huntington's disease and Parkinson's disease), and (3) prediction of time to symptom onset (presymptomatic mutation carriers in genetic Alzheimer's disease and spinocerebellar ataxia type 3).
\end{abstract}

Keywords Alzheimer's disease $\cdot$ Amyotrophic lateral sclerosis $\cdot$ Biomarkers $\cdot$ Creutzfeldt-Jakob disease $\cdot$ NFL $\cdot$ Parkinsonian syndromes

\section{Introduction}

Neurodegenerative diseases (NDDs) are currently considered as a continuum of disorders with common pathophysiological

Filippo Baldacci

filippo.baldacci@unipi.it

1 Department of Clinical and Experimental Medicine, University of Pisa, Pisa, Italy

2 Centre National de Référence des Démences Rares ou Précoces, IM2A, Département de Neurologie, AP-HP - Hôpital Pitié-Salpêtrière, Paris, France

3 FrontLab, Institut du Cerveau et de la Moelle épinière (ICM), Paris, France

$4 \mathrm{GRC} \mathrm{n}^{\circ}$ 21, Alzheimer Precision Medicine (APM), AP-HP, Pitié-Salpêtrière Hospital, Sorbonne University, Boulevard de l'hôpital, Paris, France

5 INSERM U 1127, CNRS UMR 7225, Brain \& Spine Institute (ICM), Boulevard de l'hôpital, Paris, France

6 Department of Neurology, Institute of Memory and Alzheimer's Disease (IM2A), Pitié-Salpêtrière Hospital, Paris, France mechanisms, including misfolded protein deposition, neuronal synaptic disruption, axonal degeneration, neuroinflammation, and oxidative stress [1-3]. Therefore, the greatest current challenge in the field of NDDs is to provide biomarkers for the pathological mechanisms underlying each clinical picture [4], in order to improve the diagnostic and prognostic stratification of the patients and to allow early diagnosis and disease monitoring as well as to test treatment efficacy.

Within this multifaceted scenario, neurofilament light chain (NFL) is, at the present, the most promising candidate biomarker for an early identification of a general neurodegenerative process able to support disease diagnosis, prognosis, and progression, as well as monitoring an eventual disease-modifying treatment [5-7]. It is a component of neurofilaments (NFs) that, together with glial filaments, are the main types of intermediate filaments (IFs) of the nervous system [9-11]. Its physiological function is to confer mechanical stress resistance by preserving the characteristic cellular shape, intracellular traffic regulation between axons and dendrites, and, indirectly, nerve conduction speed modulation maintaining axon diameter [11]. Recent research suggests that they are also important for normal synaptic function [12]. Axonal dysfunction and degeneration are 
important steps in NDD pathogenesis, occurring long before neuronal cell death and often preceding detectable deposition of misfolded proteins [8]. During these processes, NFL is released into the extracellular space and, consequently, into body fluids, such as the cerebrospinal fluid (CSF) and blood.

Broadly speaking, mounting data reported increased CSF NFL levels in NDDs [5, 6, 13]. In the mid-1990s, through the first enzyme-linked immunosorbent assay (ELISA) developed for NFL, Rosengren and colleagues [14] demonstrated that CSF NFL concentration was increased in amyotrophic lateral sclerosis (ALS), Alzheimer's disease (AD), and vascular cognitive impairment (VCI). Although with different magnitudes, further studies revealed that CSF NFL increased also in other several NDDs, such as frontotemporal dementia (FTD) [15, 16], Parkinson's disease (PD) and atypical parkinsonisms (APs) [17, 18], Huntington's disease (HD) [19], mild cognitive impairment (MCI) [20, 21], and Creutzfeldt-Jakob disease (CJD) [22, 23], as well as in non-primary neurodegenerative disorders, such as multiple sclerosis (MS) [24], neuroinfectious conditions [25], traumatic brain injury [26], acute stroke [27], and cerebrovascular diseases [28]. Moreover, in NDDs, CSF NFL levels showed to correlate with poorer cognition, short survival times, brain atrophy, and disease severity and progression [29-31], supporting the notion that it could be useful not only as a diagnostic biomarker but also as a prognostic and progression biomarker [32]. As a result, it has been proposed as a dynamic biomarker for axonal degeneration $[5,6,13]$ with the potential capacity to monitor treatment effectiveness [10, 33].

In the last few years, the interest in NFL research shifted toward blood. An ideal biomarker should be easily measurable, accurate, quantitative, reproducible, and employable to exactly categorize the population in line with a certain disease $[34,35]$. To this end, blood-based biomarkers would have significant advantages in time efficiency and cost efficiency compared to CSF and neuroimaging [36, 37]. Moreover, they would offer potential applications at the population level as screening tools in primary care, as well as for longitudinal evaluations with repeated sampling during follow-up. It is not surprising that brain pathophysiological processes are reflected into the periphery. However, CSF proteins partially enters the blood flow, are subsequently diluted in a greater volume compared with CSF, and go through biochemical interactions with a large amount of plasma proteins. They are also cleared by blood cells and metabolized by other tissues. Finally, these processes overall hamper their measurement in plasma or serum using traditional techniques. Nevertheless, in the past few years, the development of analytical tools for ultrasensitive quantification - the immunomagnetic reduction (IMR) and the single molecule array (Simoa) techniques - by allowing an efficient measurement of NFL in blood [38], charted a tight correlation between CSF and blood NFL in different NDDs [39]. Therefore, blood NFL was suggested as a proxy of any neurodegenerative process, paving the way to its use in clinical practice as a reliable risk biomarker for neurodegeneration $[40,41]$. Nonetheless, its potential application in real life remains unclear [42].

Biomarker is defined as "a characteristic that is objectively measured and evaluated as an indicator of normal biologic and pathogenic processes, or pharmacologic responses to a therapeutic intervention" [43]. From a clinical perspective, a biomarker can be also classified in further categories with some practical and conceptual overlaps: (1) antecedent biomarkers identifying a risk of disease development (risk biomarkers), (2) early biomarkers screening a subclinical condition (screening biomarkers), (3) biomarkers specifically recognizing a full-blown clinical picture (diagnostic biomarkers), (4) biomarkers categorizing disease severity (staging biomarkers), (5) biomarkers predicting future disease course (prognostic biomarkers), and (6) biomarkers predicting treatment response (predicting or monitoring biomarkers) [44]. Accordingly, it is crucial to define the context of use of a certain biomarker (primary care screening, diagnostic, risk of progression, disease monitoring, stratification for clinical trials, and pharmacodynamic or treatment response monitoring).

This review will attempt to summarize the current literature on blood (plasma or serum) NFL in NDDs, trying to translate research data in practical considerations, focusing on the context of use of blood NFL as a biomarker in the framework of the NDDs (Table 1).

\section{Literature Research Methods}

We conducted a systematic review of the literature until February 2020, using the key terms "NFL," "neurofilament light chain," and "neurofilament" to interrogate the PubMed database for articles published in English evaluating blood NFL concentrations (serum and plasma) in NDDs. Overall, we identified 38 studies. The use of internationally accepted clinical diagnostic criteria for each NDD, in particular AD [45-49], ALS [50-52], dementia with Lewy bodies (DLB) [53-55], FTD [56-58], PD [59-61], AP [62-65], and sporadic Creutzfeldt-Jakob disease (sCJD) [66, 67], has been checked out for any single study. The diagnostic performance of blood NFL concentrations to correctly allocate the participants to the different diagnostic groups was considered as follows: "excellent" (area under the ROC curve (AUROC) 0.90-1.00), "good" (AUROC 0.80-0.89), "fair" (AUROC 0.70-0.79), "poor" (AUROC 0.60-0.69), or "fail” (i.e., no discriminatory capacity) (AUROC 0.50-0.59) [68].

$\mathrm{AD}$ is the most common form of dementia in the elderly, accounting for $50-70 \%$ of prevalent neurodegenerative 151

.

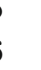

37

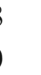

(

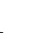

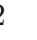

.

5

6

8


Mol Neurobiol

t1.1 Table 1 Overview on the

t1.2 possible context of use of blood

$\mathrm{t} 1.3$

$\mathrm{t} 1.4$

$\mathrm{t} 1.5$

$\mathrm{t} 1.6$

$\mathrm{t} 1.7$

$\mathrm{t} 1.8$

t1.9

$\mathrm{t} 1.10$

$\mathrm{t} 1.11$

$\mathrm{t} 1.12$

t1.13

$\mathrm{t} 1.14$
NFL as a biomarker in NDDs

\begin{tabular}{|c|c|c|c|c|c|}
\hline & \multicolumn{3}{|c|}{ Diagnostic value } & \multirow{2}{*}{$\begin{array}{l}\text { Prognostic } \\
\text { value }\end{array}$} & \multirow{2}{*}{$\begin{array}{l}\text { Monitoring } \\
\text { treatment }\end{array}$} \\
\hline & $\begin{array}{l}\text { Preclinical } \\
\text { phase }\end{array}$ & $\begin{array}{l}\text { Prodromal } \\
\text { phase }\end{array}$ & $\begin{array}{l}\text { Full-blown } \\
\text { picture }\end{array}$ & & \\
\hline $\mathrm{AD}$ & \pm & + & + & + & \pm \\
\hline PD & \pm & \pm & + & + & \pm \\
\hline $\begin{array}{l}\text { Atypical parkinsonisms } \\
\text { (4R tauopathies) }\end{array}$ & \pm & \pm & + & + & \pm \\
\hline DLB & \pm & \pm & \pm & \pm & \pm \\
\hline FTD & - & \pm & + & \pm & \pm \\
\hline ALS & \pm & \pm & + & \pm & \pm \\
\hline CJD & \pm & + & + & - & \pm \\
\hline HD & - & - & - & + & \pm \\
\hline SMA & - & - & - & \pm & + \\
\hline Sporadic late-onset ataxias & \pm & \pm & \pm & + & \pm \\
\hline NDDs as a whole & \pm & + & + & + & \pm \\
\hline
\end{tabular}

Plus sign (+), potential use, supportive data are available; plus-minus sign $( \pm)$, unknown; negative sign, negative evidences are available

$A D$ Alzheimer's disease, $A L S$ amyotrophic lateral sclerosis, $C J D$ Creutzfeldt-Jakob disease, $D L B$ dementia with Lewy body, FTD frontotemporal dementia, $H D$ Huntington's disease, $N D D$ neurodegenerative diseases, $P D$ Parkinson's disease, SMA spinal muscular atrophy dementia cases with an enormous health and economic impact [69]. The scientific and clinical research is nowadays shifting from dementia to the prodromal or even preclinical phases of $\mathrm{AD}$ to find effective therapeutic interventions that can delay or halt neurodegenerative progression [70, 71].

Biomarkers hold promise for improving early diagnosis in $\mathrm{AD}$ and establishing a tailored approach. The use of specific surrogate biomarkers (neuroimaging, blood [plasma/serum], and $\mathrm{CSF}$ ) of $\mathrm{AD}$ pathology has been included in revised diagnostic criteria to distinguish $\mathrm{AD}$ from other forms of dementia since its early disease stages. However, postmortem studies demonstrate a high degree of neuropathologic heterogeneity in patients who received a clinical diagnosis of $\mathrm{AD}$ [72]. The pathogenesis of $\mathrm{AD}$ involves interacting pathophysiological cascades in which the deposition of amyloid plaques $(A \beta)$ and the formation of neurofibrillary tangles (NFTs) composed of hyperphosphorylated tau protein would represent only the core events. The recently established " $\mathrm{A} / \mathrm{T} / \mathrm{N}$ " scheme proposes three binary biomarker categories which reflect $A D$ pathophysiology, where " $A$ " refers to $A \beta$ pathology, "T" to tau pathology, and $\mathrm{N}$ to neurodegeneration $[73,74]$. However, emerging evidence stresses the existence of additional molecular pathophysiological pathways, such as synaptic dysfunction and degeneration, innate immune response and neuroinflammation, vascular and cell membrane dysregulation, brain metabolic dysfunction, and axonal disruption [75]. The latter is prominent in $\mathrm{AD}$, and it is more closely related to cognitive decline than $\mathrm{A} \beta$ pathology [76], thus leading to propose CSF NFL as a non-specific biomarker to detect early AD pathophysiological alterations [77]. In addition, an increased release of NFL molecules is a consequence of aging that contributes to an axonal degeneration due to subclinical cerebrovascular changes and neuronal atrophy [78]. In this regard, a recent prospective community-based study enrolling a cohort of cognitively intact subjects reported high variability of serum NFL levels above 60 years [79].

Several studies showed that CSF NFL levels are elevated in $\mathrm{AD}$ patients when compared with healthy controls (HCs) and higher NFL concentration is predictive of a rapid disease progression along core biomarkers of AD pathology [21]. Peripheral serum or plasma NFL strongly correlated with CSF NFL concentration, suggesting that it reflects the same pathological process [80]. In general, we found that the levels of NFL are higher in serum than in plasma, but the majority of studies used plasma to quantify NFL.

\section{NFL as Diagnostic Biomarker}

Current evidence revealed that plasma NFL allows to discriminate $\mathrm{AD}$ patients from $\mathrm{HC}$ subjects with a good/excellent diagnostic accuracy [77, 81] (Table 2). In addition, plasma NFL levels showed to be higher in the AD dementia group than in the MCI group and in $\mathrm{A} \beta$-positive MCI patients than $\mathrm{HC}$ [77]. More recently, this finding has been replicated in a larger study in the Dominantly Inherited Alzheimer Network (DIAN) [82]. Other studies reported higher plasma/serum NFL levels in AD and MCI patients compared with controls $[76,83,84]$, but with conflicting results about the differences between MCI and cognitively normal individuals. A recent meta-analysis by Wang and colleagues [85] confirmed these findings, supporting a possible contribute of plasma NFL in the AD diagnostic workup. Moreover, plasma NFL levels could also reflect NFT pathology (as determined by NFL immunostaining) and neurodegeneration at postmortem evaluation [76]. 


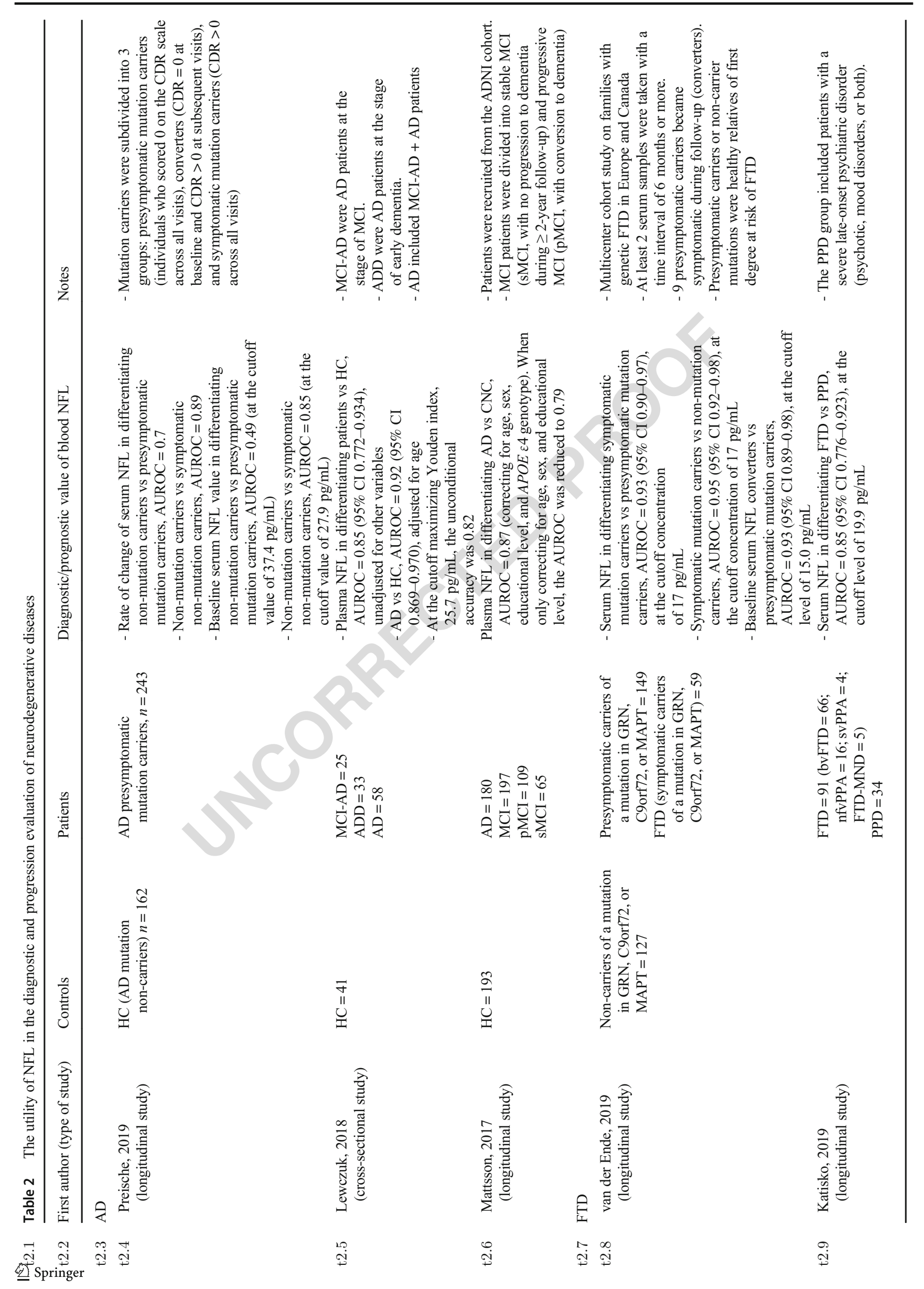




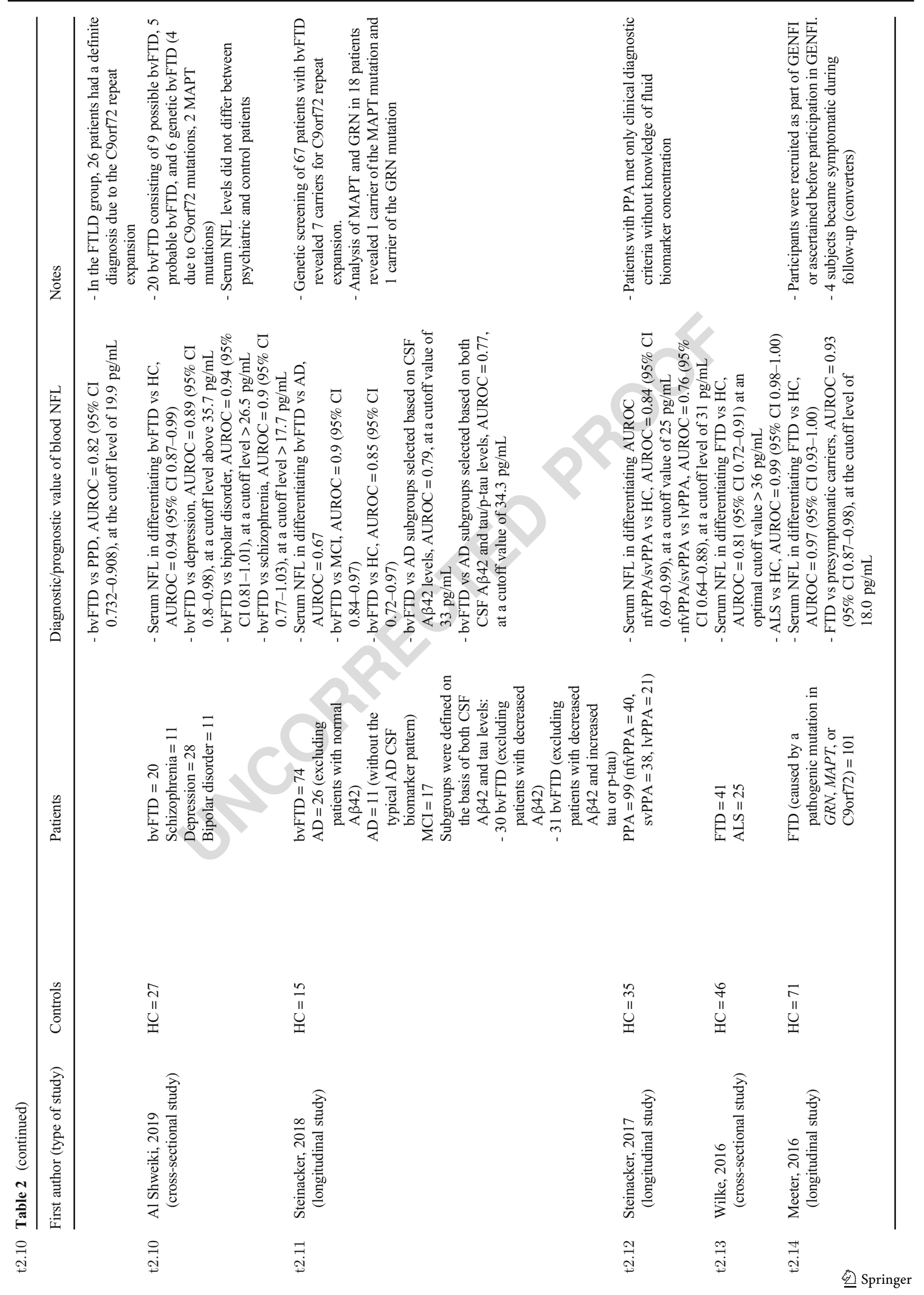



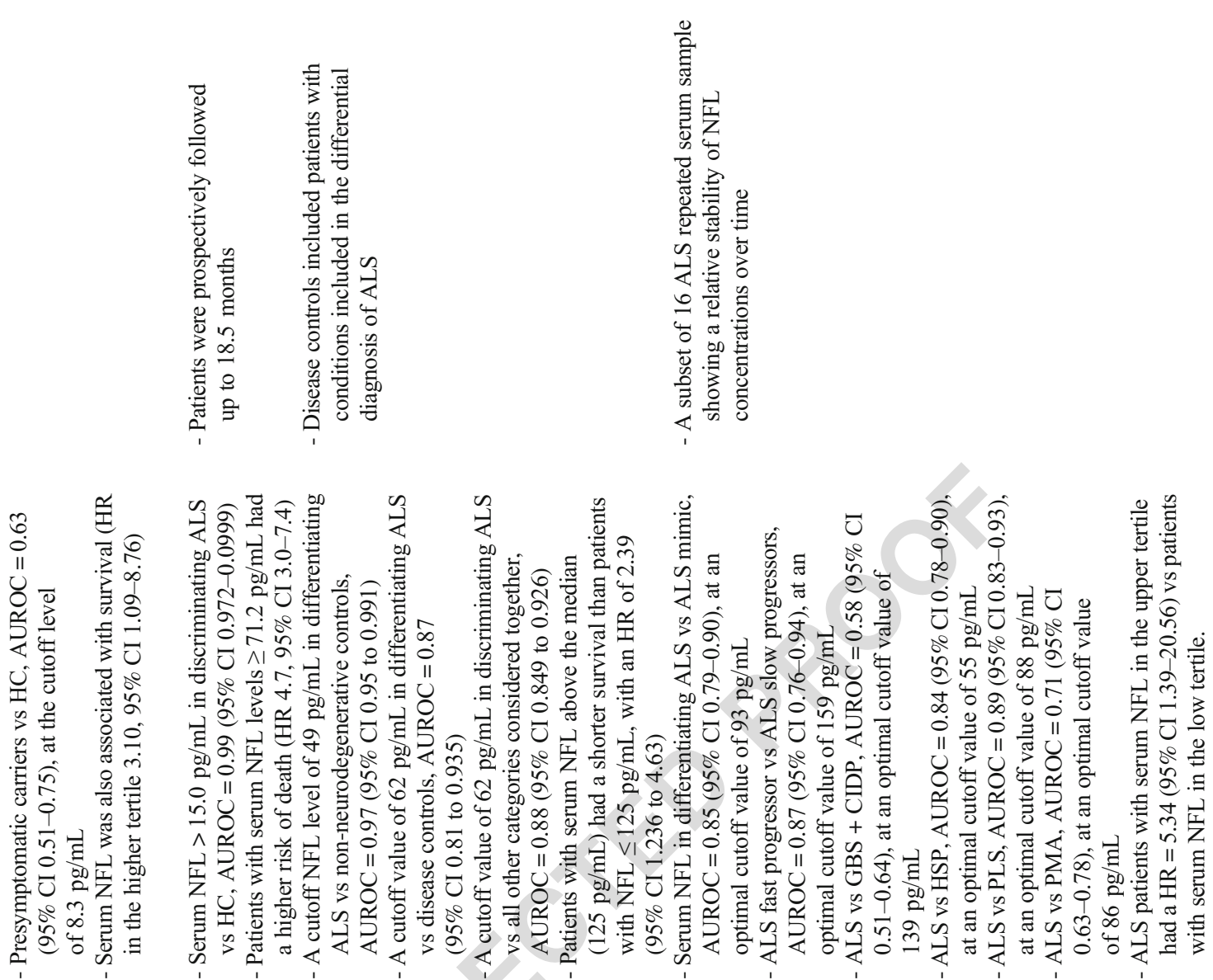

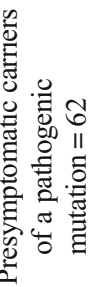
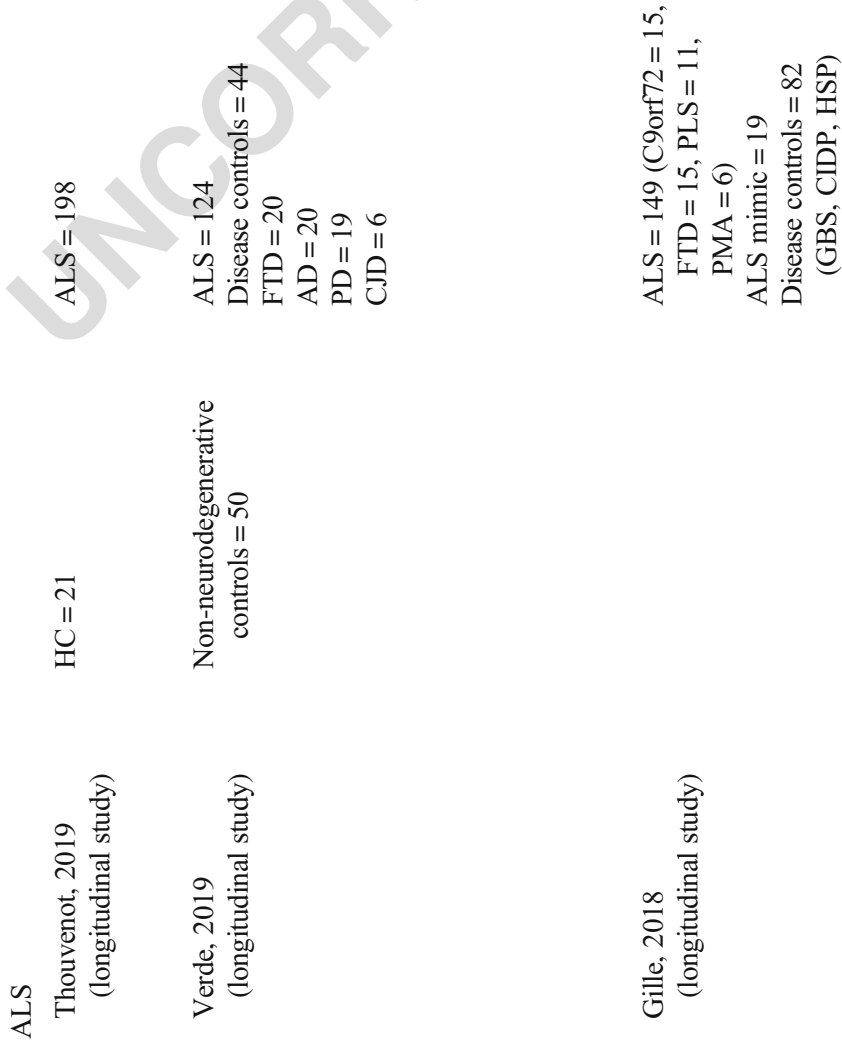

id

공 


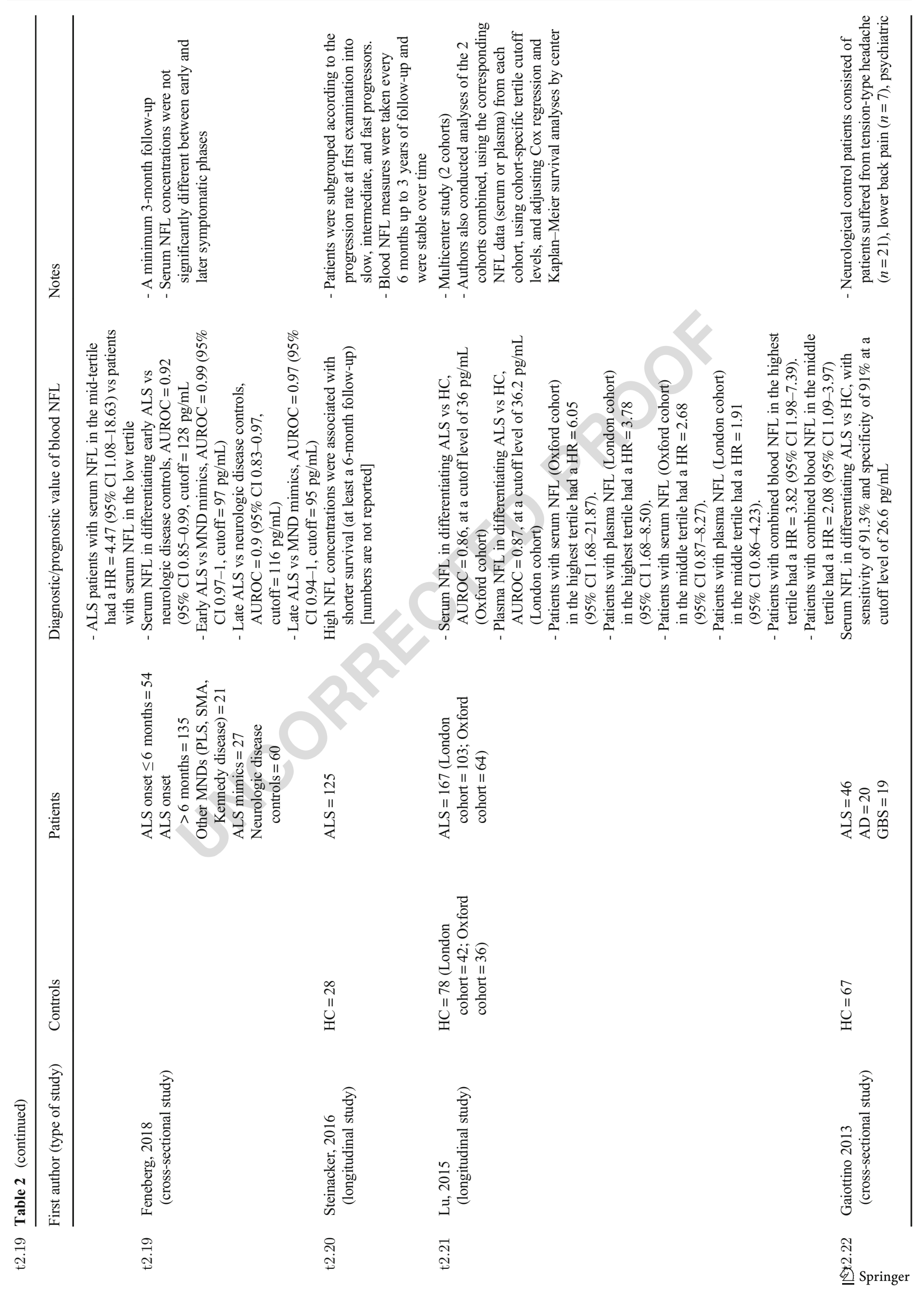




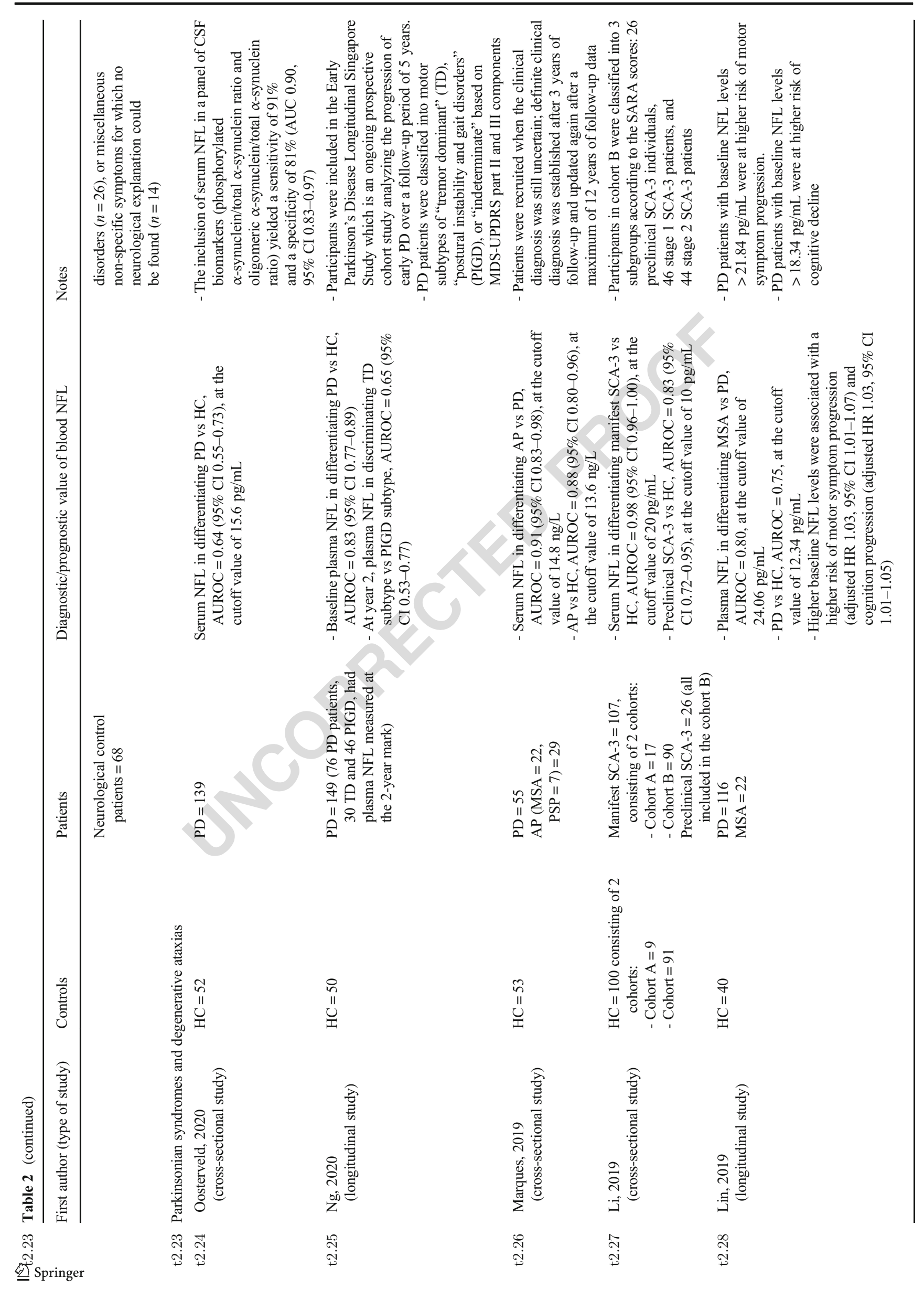




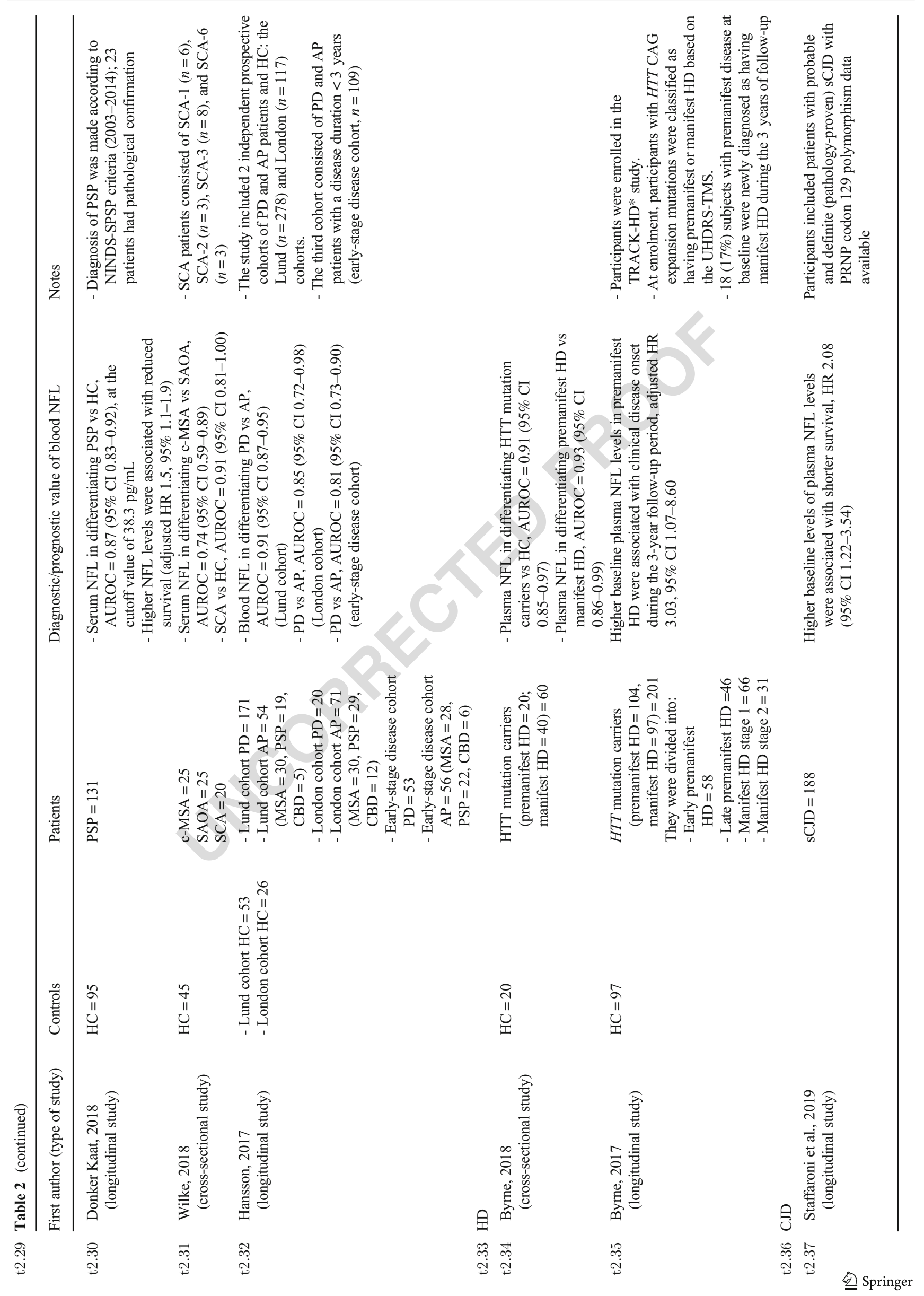




\section{AU'Tī}

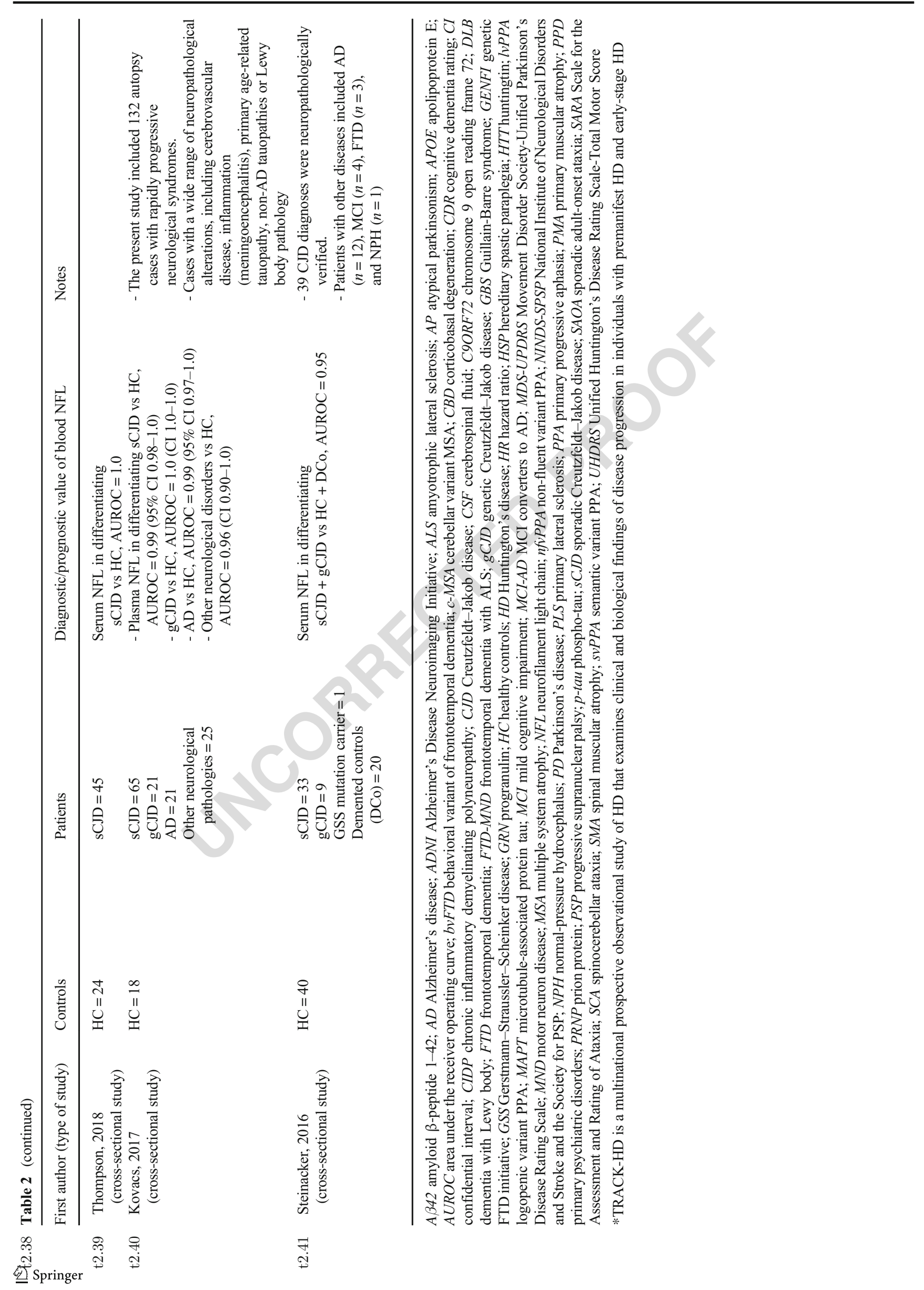




\section{NFL as Staging and Prognostic Biomarker}

Studies in AD and MCI-AD patients found a correlation between plasma NFL concentration and cognitive impairment, MRI hippocampal volume loss and brain atrophy, and cerebral ${ }^{18 \mathrm{~F}}$ FDG-PET hypometabolism [76, 81-87]. Moreover, higher plasma NFL levels predicted faster cognitive deterioration and a higher rate of brain atrophy and hypometabolism in MCI patients over time [77]. Baseline plasma and CSF NFL levels were similarly associated with short-term declines in imaging measures of neurodegeneration and with global cognitive worsening, but not with change in amyloid ligand retention on PET [35], differently from CSF t-tau concentration that critically depends on cerebral A $\beta$ burden [88]. Instead, increased plasma NFL was related to baseline and longitudinal glucose hypometabolism, which is an unspecific neurodegeneration marker, in ADrelated regions of MCI A $\beta+$ individuals [87].

In a longitudinal analysis of NFL plasma levels in a large cohort of subjects enrolled in the Alzheimer's Disease Neuroimaging Initiative (ADNI), Mattsson and colleagues [82] found increasing rates of NFL changes from preclinical $\mathrm{AD}$ stage to frank $\mathrm{AD}$ dementia through prodromal phase, suggesting NFL as a dynamic biomarker sensitive to AD disease progression. Of note, longitudinal NFL variations correlated with several baseline AD-related features (CSF biomarkers, imaging measures, and cognition) in the whole population, though with significant differences regarding clinical stage. Actually, the strictest associations were reported in MCI participants. Longitudinal NFL level was generally increased in patients who were classified as $\mathrm{N}+$ (using temporal brain atrophy as $\mathrm{N}$ - indicator) and in those who were only $\mathrm{T}+$. Therefore, NFL might reflect a neurodegenerative process that occurred independently from $A \beta$ pathology. Noteworthy, the NFL rate of change, rather than NFL absolute concentration, was subject to a significant increase in mutation carriers compared with non-carriers. Moreover, the NFL rate of change strongly correlated with longitudinal precuneus cortical thinning in both symptomatic and presymptomatic mutation carriers [89].

\section{NFL as Risk/Screening Biomarker}

Blood NFL levels seem to predict the progression to AD dementia in patients with subjective memory complaints [77]. An association between regional hypometabolism in the right hippocampus and higher plasma NFL levels was reported in cognitively normal participants from the ADNI database [87]. $\mathrm{Hu}$ and colleagues [90] explored the predictive role to develop $\mathrm{AD}$ of plasma NFL at the preclinical stage. Interestingly, plasma NFL concentrations were already abnormally high in cognitively normal individuals with significant $A \beta$-related pathological changes. Baseline plasma NFL levels did not differ in normal elderly volunteers who remain cognitively intact during the follow-up, independently from an initial amyloid PET positivity status. Instead, a trend toward elevated plasma NFL concentration was observed in $A \beta+$ individuals with subjective memory complaints compared to subjects without memory complaints who were $\mathrm{A} \beta+$, and plasma NFL resulted to be inversely associated with cognitive performance [91]. By contrast, other groups investigated the correlation between serum NFL levels with cerebral metabolism in MCI patients. Regional hypometabolism in bilateral parahippocampal gyri, right fusiform, and middle temporal gyri was independently predicted by plasma NFL [92].

Weston and colleagues [93] reported increased serum NFL concentrations also in symptomatic and presymptomatic familial AD (FAD) mutation carriers, showing a significant correlation with the estimated years to/from symptom onset across all mutation carriers as well as with cognitive decline and MRI atrophy. This finding suggests that increases in serum NFL precede the onset of AD symptoms. A large study in the DIAN cohort confirmed NFL as a sensitive marker of early neurodegeneration, finding significant increased serum NFL levels in AD mutation carriers (A $\beta$ precursor protein (APP) or presenilin 1 (PSEN1) or presenilin 2 (PSEN2)) 16 years before disease onset [89]. The rate of change of serum NFL peaked in mutation carriers during the conversion phase to clinically evident cognitive impairment and reached a plateau in symptomatic carriers; absolute values of NFL showed a trend toward a slow increase over time (Table 2). Interestingly, the increase in plasma NFL concentration during the follow-up (15-30 months) in 79 elderly participants without dementia, including 15 subjects with MCI, was associated with a significant decline in both attention and global cognition and with an increase in cerebral amyloid PET uptake [32]. More recently, baseline serum NFL was shown to be a strong and independent predictor of brain volume loss and subtle cognitive changes in a longitudinal study cohort of neurologically intact individuals [79].

\section{NFL as Predictive Biomarker}

Although not yet in humans, transgenic mice models treated with a $\beta$-secretase (BACE) inhibitor showed beneficial effects on AD-relevant downstream markers, including reduced plasma NFL concentrations [30].

\section{FTD}

The term FTD indicates a heterogeneous spectrum of NDDs inexorably conveying to a dementia syndrome characterized by predominant behavioral—behavioral FTD (bvFTD) [94]—or language - primary progressive aphasia (PPA) [58] 
314 impairment. It is the third most common neurodegenerative 315 dementia after AD and DLB and is typically diagnosed in mid316 dle age [95]. bvFTD is the most prevalent phenotype (55-60\% 317 of cases) whereas PPA (40-45\% of cases) can be further clas318 sified as non-fluent/agrammatic variant (nfvFTD) and semantic 319 variant (svFTD) [96]. Notably, an additional clinical PPA var320 iant, named logopenic variant, can show AD pathological features in more than half of subjects [97]. Finally, a clinical overlap between FTD and ALS is described and about 10-15\% of cases with ALS report a dementia syndrome in the FTD spectrum (ALS-FTD) [98]. The underlying pathologies in FTD, essentially abnormal accumulations of either tau or TAR DNA-binding protein 43 (TDP-43) proteins, may be recognized only on postmortem examination. These misfolded protein aggregates lead to atrophy (structural MRI) and hypometabolism ( $\left.{ }^{18 \mathrm{~F}} \mathrm{FDG}-\mathrm{PET}\right)$ of frontal and/or anterior temporal lobes depending on phenotype [41].

Unfortunately, with the exception of causative mutations in genetic forms (about 10\% of cases: hexanucleotide repeat expansions near the chromosome 9 open reading frame gene (C9orf72), progranulin (GRN), and microtubule-associated protein tau (MAPT) [99]), CSF poly(GP) detection in C9ORF72 expansion carriers, and decreased CSF/blood progranulin levels in GRN mutation carriers, specific pathophysiological biomarkers for FTD are completely lacking. However, the diagnosis of FTD remains very challenging, especially for behavioral variant as its diagnosis is mainly based on clinical assessment and because its symptoms show a significant overlap with primary psychiatric disorders [100].

In this context, blood NFL is a promising candidate biomarker for FTD, especially for disease differential diagnosis, monitoring, and prognosis [101, 102]. Recent evidence proved that blood and CSF levels of NFL are tightly related and significantly higher in FTD subjects than in HC [103], without gender differences [104]. Furthermore, although NFL levels generally increase with aging, this association seems not present for FTD patients [16, 105-107]. Blood NFL levels tightly correlate with CSF values in almost all studies measuring this biomarker in both fluids, thus suggesting that the peripheral concentrations of NFL substantially reflect the pathophysiological modification within CNS leading to NFL increase in CSF.

\section{NFL as Diagnostic Biomarker}

Serum NFL levels distinguished FTD patients from controls with good/optimal diagnostic accuracy (Table 2) [105, 108-110]. Interestingly, this biomarker was higher in bvFTD individuals in comparison with psychiatric patients affected by depression, schizophrenia, and bipolar disorders [106], in which bvFTD misdiagnosis is common [100]. The diagnostic accuracy to differentiate bvFTD from psychiatric disorders was above $80 \%$ (Table 2), independently from the specific psychiatric condition (mood or psychotic disorders) [106, 107].

Only one study compared both FTD and AD subjects, reporting that serum NFL levels were higher in bvFTD patients [109], and separated the two groups with a sensitivity and specificity of $93 \%$ and $61 \%$, respectively (Table 2), after a preliminary exclusion of bvFTD patients with an AD biomarker profile and clinical $\mathrm{AD}$ subjects without a core biomarker confirmation. Moreover, serum NFL concentrations seemed to be higher in nfvPPA and svPPA than lvPPA subjects [105], though with only moderate accuracy. A further study showed no significant differences between PPA subtypes [110].

Finally, although FTD individuals had higher serum NFL levels than subjects with other cognitive disorders such as AD, ALS patients reported even more elevated concentration [108]. Notably, ALS subjects present a TDP-43 pathology in about $95 \%$ of cases, suggesting a potential association between increased NFL concentration and TDP-43 pathology. Further confirmation of this hypothesis is supported by observing that FTD forms expected to be TDP-43 positive (C9ORF72 and GRN mutation carriers, or svPPA phenotype) reported higher concentration of this biomarker than FTD subtypes expected to be tau positive (MAPT mutation carriers and nfvPPA) $[16,99,104]$.

\section{NFL as Staging and Prognostic Biomarker}

In different studies, serum NFL increases overtime in FTD subjects, independently from the phenotypes, with the exception of lvPPA [105, 109]. A longitudinal Mini-Mental State Examination (MMSE) decline was correlated with baseline serum NFL levels [107]. Disease duration was not associated with NFL concentration although one study reported a poor survival in FTD subjects in the higher tertile of serum NFL levels (Table 2).

Additionally, peripheral blood NFL concentrations appeared to reflect specific regional brain atrophy related to clinical phenotypes (PPAs or bvFTD). In bvFTD patients, serum NFL was associated with a low cognitive score and a reduction in wholebrain volume and was correlated with brain atrophy, including frontal and subcortical regions [99, 104, 109, 110].

Serum NFL was correlated with baseline cognitive impairment, cognitive decline overtime, and atrophy progression of the left frontal lobe and the right middle frontal gyrus in PPA individuals and nfvPPA/svPPA subjects, respectively [105, 107]. On the other hand, although only in one study, serum NFL concentration was not related to specific hypometabolic regions on ${ }^{18 \mathrm{~F}}$ FDG-PET in PPA subjects [110].

\section{NFL as Risk/Screening Biomarker}

Noteworthy, serum NFL was higher in genetic FTD with a full-blown clinical picture but not in presymptomatic carriers 
compared to $\mathrm{HC}$, independently from the gene mutation. Moreover, it was consistently higher in converters than in non-converter carriers [104] and increased overtime in converters but not in symptomatic FTD as well as non-converters. Therefore, serum NFL could differentiate genetic FTD patients from presymptomatic carriers, with an excellent diagnostic accuracy (Table 2) [16]. By contrast, the discriminatory accuracy of the biomarker relative to presymptomatic genetic carriers and controls resulted quite poor (Table 2).

\section{ALS}

ALS is a progressive neurological disease in which upper motor neuron (UMN) and lower motor neuron (LMN) degenerate, leading to paralysis and death, typically within 35 years from symptom onset. To date, there is no definitive diagnostic test for ALS, and confirmation of diagnosis is based on clinical findings, electromyography results, and exclusion of mimics [52]. Despite efforts to increase the sensitivity of diagnostic criteria, often the diagnosis is made only after the onset of symptoms for both sporadic and familial ALS $[111,112]$. An early diagnosis would be paramount, since it was observed that the benefit of riluzole is related to its early administration.

A large body of research exists on neurochemical ALS biomarkers [113, 114], among which phosphorylated neurofilament heavy chain (pNFH) and NFL have been postulated as the most interesting candidates $[115,116]$. This is not surprising, given the axonal impairment that characterizes the disease already at the early stage, with a large release of NF in CSF [117]. Additionally, previous studies indicate cytoskeletal proteins as one of the key factors contributing to neurodegeneration in ALS [118-123]. Other evidences provide additional support that NFL aggregation is an early event in motor neuron disease [124], and that NFL is involved in the aggregation and neurotoxicity of other proteins in motor neurons [125]. At the same time, elevated NFL levels in ALS may be explained by the higher content of axonal proteins in motor neurons compared to other neuronal populations [126]. Nonetheless, several data highlighted that NFL is able to discriminate ALS patients from healthy and disease controls [14, $117,127,128]$, especially in cases with predominant UMN signs, and correlates with clinical disability [129], disease stage, progression, and/or prognosis [130, 131], probably reflecting the burden of motor neuron degeneration. Although CSF NFL remains the more robust fluid biomarker for ALS because of its directly reflecting alterations in the CNS, a high correlation between CSF and blood NFL concentrations has been reported [132]. Moreover, at odds with other neurodegenerative diseases such AD, NFL concentrations do not correlate with age in ALS individuals [133-135].

\section{NFL as Diagnostic Biomarker}

Mounting evidence reports significantly higher blood NFL levels in ALS patients when compared to controls (Table 2) [40, 135-138]. The diagnostic performance of serum NFL in discriminating ALS and non-neurodegenerative subjects showed excellent sensitivity and specificity (Table 2) [139, 140]. These findings led authors to propose the introduction of serum NFL measurement into clinical practice as supportive diagnostic tool. In addition, serum NFL showed significantly elevated concentration in ALS even at the onset of the first symptoms, confirming its potential role as a biomarker for early detection of symptomatic sporadic ALS. In this regard, serum NFL concentrations demonstrated optimal sensitivity and specificity also in distinguishing early symptomatic ALS from other neurologic diseases or motor neuron disease mimics, independently whether diagnosis was definite, probable, or possible, following the El Escorial criteria (Table 2) [134]. Interestingly, Gille and colleagues [135] reported an increase of serum NFL as a function of the number of regions (i.e., cranial, cervical) affected by UMN degeneration. Accordingly, in a MRI-based study, elevated CSF and serum NFL concentrations were significantly associated with lower diffusion tensor imaging (DTI) fractional anisotropy and increased radial diffusivity in the corticospinal tract of ALS patients, as well as with clinically UMN score burden [133]. On the other hand, previous studies have shown that NFL levels were not increased in Kennedy disease and spinal muscular atrophy (strictly LMN diseases) [128, 134]. As a consequence, a subclinical involvement of the UMN is likely in ALS patients with isolated LMN symptoms and elevated serum NFL concentration [117]. However, the neuroanatomical correlate of NFL increase is not yet clear since Verde and colleagues [139] showed a lack of association with DTIMRI measurements of the integrity of cerebral white matter tracts in the brain of ALS patients. Finally, serum NFL levels were reported relatively lower in patients with primary lateral sclerosis (PLS) and hereditary spastic paraplegia (HSP), two UMN-isolated syndromes, compared with ALS subjects, suggesting in such patients different pathophysiological processes and rates of neurodegenerative diseases [134, 135].

\section{NFL as Staging and Prognostic Biomarker}

Several studies reported that blood NFL levels correlate with disease severity parameters, such as the decline in the ALS Functional Rating Scale-Revised (ALSFRS-R) score and the ALS Milano-Torino Staging (MITOS) system score [135, 137, 140, 141].

Furthermore, serum NFL levels at recruitment or at the time of diagnosis predicted survival independently from other clinical variables and were negatively associated with disease duration (Table 2) [136, 138, 142]. Thouvenot and colleagues 
512 [140] evaluated the largest series ever of serum samples taken 513 from ALS patients, finding that NFL concentration was the 514 most important parameter related to ALS survival in multivar515 iate models (Table 2). Likewise, serum NFL concentrations in 516 the middle and high tertile were associated with an increased 517 HR compared with those of patients in the lowest tertile 518 (Table 2) [135]. Interestingly, unlike pNFH, NFL levels seem 519 to change minimally throughout the course of the disease, 520 maintaining distinct temporal profiles from controls, and a 521 steady trajectory [136]. approved for the treatment of all SMA types, with a rather dramatic impact on phenotype [146]. The levels of two additional biomarkers of neurodegeneration (CSF tau and glial fibrillary acidic protein (GFAP), an intermediate filament present in astrocytes) decreased together with CSF NFL after nusinersen administration, indicating that the neuronal and astroglia damage can be restored by nusinersen treatment [145]. Moreover, the decrease of NFL concentration was much larger than that of tau and GFAP, suggesting NFL as an early treatment response biomarker in SMA patients, helpful to select those patients will benefit to continue such an invasive treatment. Further studies with a long follow-up are needed, but these preliminary results in SMA indicated NFL as a promising marker for upcoming disease-modifying therapies in diseases beside SMA. Conversely, it is worth mentioning that the diagnostic and monitoring value of NFL in CSF and blood has not been confirmed in adolescent and adult SMA-type (SMA types 2 and 3) patients treated with nusinersen $[134,147]$. It was hypothesized that NFL release is lower in late-onset SMA than in the infantile-onset subtype. Actually, the first phenotype is characterized by a long-lasting and chronic disease course while the foster by an acute and highly aggressive onset. Additionally, subjects with infantileonset SMA report a significantly better response to nusinersen therapy when compared to individuals with adult-onset one. In this regard, the finding of normal blood levels of NFL in SMA could be used in a diagnostic panel of biochemical markers to help differentiate patients presenting with motor neuron deficits, separating SMA from ALS. Indeed, a substantial proportion of patients with SMA initially receive a diagnosis of ALS [148].

\section{Degenerative Parkinsonisms}

PD is the most common degenerative parkinsonism, with evidence of progressive loss of dopaminergic neurons in the pars compacta of the substantia nigra. Diagnostic criteria have been recently revised to improve diagnostic accuracy imaging biomarkers as supportive features [61]. However, early diagnosis and progression prediction remain challenging for physicians. Of note, the differential diagnosis between PD and AP can be difficult, mainly at the early clinical stage. Similarly, evolution of diagnostic criteria for AP improved accuracy, but misdiagnosis rates are still high [149-152]. The APs that most commonly mimic PD are progressive supranuclear palsy (PSP) and multiple system atrophy (MSA), whereas among APs, the lowest diagnostic accuracy regards the corticobasal degeneration (CBD).

Currently, biochemical biomarkers for PD and AP are an unmet need, but many CSF/serum molecules are under evaluation. CSF NFL concentration overlaps in patients with PD, PD with dementia (PDD), and DLB and are comparable with 
611 those in $\mathrm{HC}$ [31]. In contrast, it has been demonstrated that 612 CSF NFL levels are markedly increased in AP patients. 613 Accordingly, it might discriminate between PD and AP with 614 a high degree of diagnostic accuracy [17, 18, 153, 154]. This 615 is in line with the remarkable axonal degeneration of large 616 myelinated axons occurring in AP as well as with the rapid 617 neuronal loss in such conditions [155]. Furthermore, CSF 618 NFL concentration correlates with measures of disease sever619 ity and other clinical variables, demonstrating its capability to 620 reflect neurodegenerative mechanisms. However, to over621 come the well-known limits related to CSF examination, 622 blood-derived NFL would be a more favorable biomarker. 623 In this regard, the strong correlation between blood and CSF 624 NFL levels in parkinsonian syndromes holds potential for an 625 application in clinical practice [156].

\section{PD}

\section{NFL as Diagnostic Biomarker}

Similar to CSF results, NFL concentration in serum/plasma is considered useful for a differential diagnosis between PD and AP [157]. This has been tested for the first time in three independent prospective cohorts of $\mathrm{PD}$, PSP, MSA, and CBD patients, compared with HC. Blood NFL levels in AP were significantly elevated compared with those in $\mathrm{PD}$, showing a diagnostic accuracy ranging from good (in the early cohort with disease duration $<3$ years) to excellent (in the Lund cohort) (Table 2) [156]. Conversely, blood NFL levels were not able to accurately separate PD from HC. A subsequent study in subjects with an uncertain diagnosis at the time of inclusion confirmed similar results (Table 2) [158]. Nevertheless, recent studies support the promise of plasma NFL as a diagnostic biomarker also in $\mathrm{PD}$, demonstrating relatively higher NFL levels in cases vs controls [159] and a good diagnostic accuracy in differentiating PD patients from HC (Table 2) [160]. Moreover, higher serum NFL levels were found even at early stages of the disease and in participants at risk of disease progression (prodromal PD and symptomatic and asymptomatic mutation carriers of known PD genetic mutations), indicating the presence of active disease and potential for conversion to either PD or parkinsonian syndromes [161].

\section{NFL as Staging and Prognostic Biomarker}

Heterogeneous results regarding possible correlations between blood NFL and PD clinical features in three independent PD cohorts have been provided in the prospective and longitudinal study of Hansson and colleagues [156]. In general, higher blood NFL levels were observed in more advanced PD and, in the Lund PD cohort, a higher blood NFL concentration was associated with disease duration and more severe motor symptoms (measured as Hoehn and Yahr (H\&Y) stage, Unified Parkinson's Disease Rating Scale (UPDRS) III motor score, Timed Up and Go Test, and Tandem Gait Test). Conversely, no clinical correlations were described in the London cohort and the early-stage disease cohort [156]. However, further studies confirmed the positive relation between plasma NFL levels and motor symptom severity (measured as H\&Y stage and UPDRS part III score) and proved a significant correlation between plasma NFL concentration and cognitive dysfunction at MMSE [161, 162]. In another study, PDD patients reported higher plasma NFL level compared with PD subjects without dementia [84], supporting an association between plasma NFL and cognitive function in PD patients [84]. Furthermore, higher baseline plasma NFL concentrations in PD patients were found to be longitudinally associated with a higher risk of progression for both motor and cognitive symptoms, suggesting that serum NFL may be a biomarker of clinical progression in PD (Table 2) [160-162].

PSP

\section{NFL as Diagnostic Biomarker}

Two other studies confirmed the diagnostic value of blood NFL in PSP patients showing good capability to discriminate between PSP and HC (Table 2) [163, 164]. In contrast, blood NFL is not suitable to separate PSP from other forms of AP [156] and similar levels are reported in patients with MSA and PSP [158].

\section{NFL as Staging and Prognostic Biomarker}

Greater baseline NFL levels in serum/plasma seem to correlate with disease severity and clinical progression in PSP patients, though with conflicting results. Such heterogeneity may reflect differences in study design, since PSP patients have been evaluated as a separate group in some studies but not in others where PSP, MSA, and CBD patients have been combined as a whole group. Specifically, blood NFL levels positively correlated with motor symptom severity, evaluated as H\&Y stage and with UPDRS III motor score, but not with disease duration or other clinical assessments, in the AP group (including also MSA and CBD patients) [156]. Similarly, serum NFL concentration at baseline correlated with motor performances, measured with the International Cooperative Ataxia Rating Scale score and Tandem Gait Test in another cohort of AP patients including PSP [158].

In studies focusing exclusively on PSP patients, higher serum NFL levels were related to more severe motor, functional, and cognitive disability as well as shorter survival but not with age at symptom onset or disease duration (Table 2) [163]. Notably, NFL levels in the higher tertile were 
associated with worse survival (Table 2) [164]. Higher baseline plasma NFL levels also predicted greater whole-brain and superior cerebellar peduncle volume loss at 1-year follow-up [163].

\section{MSA and Degenerative Ataxias}

\section{NFL as Diagnostic Biomarker}

As for PSP, studies indicate elevated blood NFL concentration in MSA patients, suggesting its use in discrimination of MSA from PD and HC with a good diagnostic accuracy (Table 2). However, as aforementioned, NFL cannot discriminate among APs [158].

Moreover, blood NFL was proposed to improve the differential diagnosis of degenerative ataxias. In a pilot study evaluating serum NFL levels in patients with a clinical diagnosis of probable cerebellar-MSA (c-MSA) subtype, sporadic adultonset ataxia (SAOA), and frequent repeat-expansion spinocerebellar ataxias (SCAs 1, 2, 3, and 6) and in HCs, serum NFL concentration was found to be higher in SCA patients and in the c-MSA group compared with controls. This is probably the result of the diffuse involvement of spinocerebellar and corticospinal tracts in these multisystemic neurodegenerative ataxias [165]. However, the performance of serum NFL differentiating c-MSA from SAOA was only moderate (Table 2), in contrast with a higher accuracy previously reported for CSF NFL (AUC = 0.93) [166]. NFL levels were significantly lower in SAOA and comparable with those of HC. A further study investigated serum NFL concentration in large cohorts of SCA-3 subjects and demonstrated higher levels in both preclinical and manifest SCA-3 individuals compared with HC [167]. Serum NFL levels discriminated manifest SCA-3 from HC with excellent accuracy, and the diagnostic performance remained good in distinguishing preclinical SCA-3 subjects from HC (Table 2) [167]. Recently, plasma NFL concentrations resulted higher also in patients affected by Friedreich's ataxia (FA), which is the most common autosomal recessive ataxia caused by CAG repeat expansion in the ATXN3/MJD1 gene, compared with agedmatched controls [168].

\section{NFL as Staging and Prognostic Biomarker}

Serum NFL in c-MSA patients does not seem to correlate with clinical disease severity (as assessed by the Scale for the Assessment and Rating of Ataxia (SARA)) or disease progression [165]. Similarly, a recent study in 99 patients with genetically confirmed FA did not find a correlation with disease severity (as defined by SARA score), age at onset, or disease duration [169]. Moreover, serum NFL concentration remains stable in a subgroup of $14 \mathrm{FA}$ patients who received a 2-year follow-up evaluation [169].

Conversely, serum NFL concentration increased with disease severity in a large cohort of SCA-3 patients, including manifest and preclinical individuals, and correlated with both clinical scales (according to SARA and International Cooperative Ataxia Rating Scale (ICARS) scores) and reduction of cerebellar and brainstem volume [167]. Preclinical SCA-3 group was divided in early and late preclinical subgroups using the median predicted number of years to onset of manifest disease. Serum NFL concentrations resulted higher in manifest than preclinical SCA-3 subjects and in late preclinical SCA-3 subjects compared with early preclinical SCA3 individuals. However, no differences were observed between early preclinical subjects and HC [167]. Despite CAG repeat count is a well-known prognostic factor for SCA-3 and FA, a correlation between serum NFL and CAG repeat lengths has been inconsistently reported $[168,169]$.

\section{NFL as Risk/Screening Biomarker}

In their study on 133 SCA-3 patients, Li and colleagues [167] demonstrated higher serum NFL concentrations in 26 preclinical ATXN3 mutation carriers (patients with SARA score $<3$ ) compared with controls. Moreover, a correlation between motor symptoms, neuroimaging markers, and serum NFL was found in all ATXN3 mutation carriers, suggesting that NFL may serve to track neurodegeneration and disease progression already in pre and prodromal SCA-3 phases.

\section{HD}

Among the most common neurodegenerative diseases, HD is unique, since the major part $(\approx 99 \%)$ of individuals presenting a HD phenotype have a mutation in the same gene [170]. Indeed, HD is an autosomal dominant inherited neurodegenerative disease with the typical manifestations of involuntary movements, psychiatric symptoms, and cognitive decline. The etiological basis is the deleterious expansion of polyglutamine encoding CAG repeats in the huntingtin (HTT) gene, leading to the expression of neurotoxic mutant huntingtin (mHTT) and extensive degeneration of neurons primarily occurring in the striatum and cortex [171]. The disease usually starts in midlife, with age of onset inversely correlating to CAG repeat number [171]. Although the cause is known, disease-modifying treatments are not yet available. In $\mathrm{HD}$, a reliable genetic test confirms a clinical diagnosis in symptomatic people or predicts disease onset in asymptomatic mutation carriers [172]. As a consequence, a novel biomarker should be directed to track disease progression and predict a treatment response to targeted therapies. Although not conclusive, the results of the available studies display that blood NFL 
Mol Neurobiol

could have a role in this context. Previous reports on CSF NFL indicate elevated concentrations in HD subjects [19, $173,174]$. This is not surprising because mtHTT interacts with other proteins altering their function and finally leading to abnormal protein aggregation and impaired axonal transport [175]. Furthermore, the level of misfolded mtHTT protein correlates with NFL concentration in CSF, thus suggesting a contemporary releasing of both proteins from damaged neurons [176].

\section{NFL as Staging and Prognostic Biomarker}

In the first retrospective study investigating NFL concentrations in the blood of premanifest HD (preHD) and early-stage HD patients enrolled in the TRACK-HD cohort, Byrne and colleagues [177] showed higher baseline NFL levels in 201 HTT mutation carriers, including 58 with early premanifest and 46 subjects with late premanifest disease, than in controls. Moreover, NFL concentration reflected baseline motor and cognitive deficits in HD patients and differed significantly with increasing disease stage. Positive associations were found between plasma NFL concentration, age, and CAG triplet repeat counts, with higher CAG lengths being associated with earlier and steeper increases in plasma NFL [177]. Therefore, NFL is the first biofluid marker showing a direct relationship with a causative gene expansion [178].

Of note, baseline plasma NFL predicted rates of brain atrophy, cognitive decline, and worsening of functional ability and motor performance in mtHTT carriers [177]. Interestingly, it was closely associated with the rate of whole-brain atrophy than with the rate of striatal one, suggesting that plasma NFL reflects more the rate of global neuronal degeneration than that of a specific brain area [177]. Additionally, in the same TRACK-HD cohort, Johnson and colleagues [179] showed voxel-wise region-specific associations between plasma NFL levels and both cross-sectional and longitudinal MRI cortical thinning and white matter volume reduction, highlighting the value of NFL as a dynamic and robust marker of brain atrophy. Notably, higher concentrations of NFL in plasma were associated with lower volume in regions known to be affected in HD and predicted subsequent occipital gray matter atrophy and widespread white matter reduction over the 3-year follow-up, independently of age and CAG length repeats [179]. NFL increased significantly from baseline both in individuals with premanifest HD and in those with manifest HD [179]. Remarkably, in a subsequent study, Byrne and colleagues [180], combining CSF/plasma NFL and CSF mutant huntingtin protein (mHTTp) as biofluid biomarkers, demonstrated that NFL levels were more accurate than mHTTp to discriminate between premanifest and manifest HDs and correlated with severity of symptoms better than mHTTp in manifest HD.

\section{CJD}

CJD is the most common human prion disease. Approximately $85 \%$ of cases are SCJD, but in a minority of cases, CJD can be genetically determined (gCJD) [181]. The disease is a rapidly progressive and fatal neurodegenerative condition, whose different phenotypes depend, at least in part, by polymorphisms on the gene encoding prion protein (PrP) [182]. Diagnosis is frequently tardive and relies on clinical World Health Organization (WHO) criteria supported by detection of the 14-3-3 protein and, more recently, t-tau in the CSF [66, 183, 184]. Also, CSF NFL recently demonstrated to be a reliable biomarker in the CJD diagnostic workup. Although few studies explored its role as a biomarker in CJD patients so far, CSF NFL levels are significantly increased in CJD (including those with more slowly progressive and atypical disease course) compared with $\mathrm{AD}$, FTD, other NDDs (dementia), and controls, indicating a massive synaptic degeneration and neuroaxonal damage in CJD [22, 23, $185,186]$. Additionally, it is noteworthy that NFL concentrations in CSF appear highly variable among different sCJD subtypes, with higher NFL levels in those with more rapidly progressing disease [187]. Importantly, plasma NFL correlates with CSF NFL concentration and recent studies suggest that blood NFL can accurately reflect the massive neurodegeneration in CJD patients.

\section{NFL as Diagnostic Biomarker}

Diagnostic accuracy of serum NFL for discrimination between CJD and controls was excellent (Table 2) [185]. These findings have been independently replicated in other two studies. Serum NFL distinguished patients from controls with $100 \%$ sensitivity and $100 \%$ specificity in 45 sCJD patients enrolled in the National Prion Monitoring Cohort [188]. Noteworthy, Kovacs and colleagues [189] reported high sensitivity and specificity of plasma NFL concentration in discriminating CJD subjects from non-CJD controls in a cohort of 132 pathologically classified patients (sCJD, gCJD, and $\mathrm{AD}$ cases) showing a rapidly progressive neurological picture. However, in this study, the diagnostic value in the differentiation between prion and other disease cases resulted lower than previously reported investigations (Table 2) [189]. Moreover, serum NFL values have been elevated since the early phases of the disease, suggesting a possible role as a screening biomarker [188]. Conversely, serum NFL concentration overlapped between ALS and CJD patients in a recent prospective study, even though the size of CJD group was very small [139].

\section{NFL as Staging and Prognostic Biomarker}

Longitudinal changes in serum tau and NFL levels were investigated in the aforementioned study of Thompson and 
colleagues [188]. However, despite a trend toward increasing concentrations of both tau and NFL over the last 12 months before death, at variance with tau, no association was found between serum NFL concentration and speed of decline on the Medical Research Council (MRC) Prion Disease Rating Scale [188]. Also, a recent study strengthens the tight association of plasma tau levels with the rate of disease progression and survival time in SCJD [190].

\section{NFL as Risk/Screening Biomarker}

gCJD forms are linked to mutations in the prion protein gene (PRNP) inherited with an autosomal dominant pattern and variable penetrance [191]. The relationship between genotype and phenotype remains a matter of debate, and index cases do not always have a family history [192]. Under this scenario, the discovery of biomarkers serving as surrogates of outcome in clinical trials may be crucial because PRNP mutation carriers might benefit from a presymptomatic intervention. PrPlowering therapeutics are now in preclinical development, and to this end, CSF total PrP has demonstrated to be a strong candidate fluid biomarker showing stable low levels in an ongoing natural history study including presymptomatic mutation carriers and normal controls [193].

\section{Conclusions}

The impact of physiological variables on blood NFL concentration, such as sex and age, has not been systematically investigated across published studies. Other variables possibly affecting its modification in peripheral blood, including systemic comorbidities and concomitant drug therapies, were not taken into account. Liver and renal clearance as well as blood cell counts and plasma protein composition could affect biomarker concentrations [194]; however, these factors were not investigated. Indeed, blood NFL concentration alterations associated with renal and hepatic dysfunctions remain unknown, thus representing a potential relevant methodological bias, especially in subjects with NDDs, being generally old and frequently exhibiting vascular comorbidities. In general, blood NFL concentrations correlate with aging due to a subtle axonal degeneration and vascular changes in elderly. However, such an association was clearly absent in CJD, ALS, and AP. This suggests that the probable contribution of aging on NFL concentrations and neurodegeneration becomes trivial in highly aggressive forms of NDDs [195].

Blood NFL concentrations are reported to be massively elevated in ALS patients, even in early disease stages, indicating its value as an efficacious, yet unspecific, biomarker in the differential diagnosis of ALS from ALS mimics. Moreover, blood NFL concentrations can reflect disease severity and/or progression in ALS, suggesting that peripheral NFL could contribute to support ALS prognosis. Other confounding variables, such as the discordant disease progression in the different clinical subtypes of ALS, should be elucidated in further studies. Actually, blood NFL could help better stratify the multifaceted clinical presentation of ALS phenotypes. However, further studies are needed to confirm its prognostic value. In contrast with genetic forms of AD, blood NFL concentrations are not increased in presymptomatic ALS mutation carriers appearing to tightly link to the symptomatic phase of the disease.

Another NDD where blood NFL increases result impressively is CJD. Because of early diagnosis remains challenging, NFL might be a reliable screening blood-based biomarker with a potentially high negative predictive value for CJD subjects, ruling out more common and less aggressive neurodegenerative dementia, such as AD. Definitely, blood NFL could be of usefulness as a first-step examination to promptly detect CJD during its prodromal phase and to start future disease-modifying treatments. On the other hand, preliminary findings do not support a potential use of NFL as a predictor of longitudinal disease progression in CJD, and its specificity should be further substantiated in comparison with other highly aggressive forms of NDD.

All reported studies showed a good or even excellent diagnostic performance of blood NFL in distinguishing patients with neurodegenerative disorders from HC. The potential contribution of this biomarker candidate to discriminate between different dementia disorders remains ambiguous given the lack of pathognomonic specificity. However, mounting data suggest that blood NFL could be a useful diagnostic tool in the diagnostic workup of FTD, to distinguish FTD (especially FTD with a TDP-43 pathology) from AD patients and to identify PPA with a likely underlying AD pathology, including lvPPA. Moreover, it can represent a biomarker tracking the disease progression and potentially identifying the transition phase from the presymptomatic to the symptomatic stage of the genetic forms of the disease. Most importantly, serum NFL is assumed to be a promising screening tool to rule out an underlying neurodegenerative disease in individuals with psychiatric disorders.

In $\mathrm{AD}$, blood NFL may predict progression to dementia in individuals with MCI at high risk and identify preclinical AD before the conversion phase. Moreover, NFL captures early neurodegenerative changes in presymptomatic familiar AD mutation carriers in which blood NFL concentrations correlate with the predicted time to symptom onset. Further studies are crucial to calculate the negative predictive value of blood NFL as a screening tool in large and selected cohorts of individuals at risk of neurodegeneration and $\mathrm{AD}$, such as individuals with subjective memory complaints and/or decline, late-onset psychiatric disorders, cerebrovascular disease, and diabetes, as well as in aging and elderly individuals in general. 
Because of the high negative predictive value of elevated blood NFL concentrations in excluding PD, this candidate biomarker can represent a valid screening tool for clinicians in the early differential diagnosis between PD and AP in cases with confounding clinical presentations. In contrast, blood NFL measurements do not suffice to differentiate PD patients from controls and cannot be used to separate PSP, MSA, and CBD from each other. Nevertheless, blood NFL may be a prognostic tool in clinical practice in both PD and AP patients. Currently, to the best of our knowledge, there are no studies investigating blood NFL in patients with DLB, and few studies are available for PDD patients.

Regarding other relevant clinical neurological presentations, blood NFL may support the classification of sporadic late-onset ataxias, notably helping in differentiating c-MSA-C from SAOA. In choreic patients, blood NFL appears to be a robust prognostic biomarker of HD disease onset and progression and holds potential as a predictive biomarker of response to disease-modifying agents in clinical trials.

Finally, blood NFL seems to be a promising candidate predictor of the timing of clinical phenoconversion in presymptomatic mutation carriers with AD, HD, and SCA-3. Conversely, blood NFL concentrations are mostly normal in premanifest ALS and FTD mutation carriers but promptly increase with the onset of clinical symptoms.

In general, given the rapid advances in elucidating the pathophysiological mechanisms of diseases, at the molecular diagnostic level, biomarkers are excellent flexible tools to improve and inform all phases of drug discovery and development by enabling validation of mechanisms of actions [196, 197]. For this reason, NFL is assumed to act as an innovative molecular mechanistic biomarker supporting in vivo detection and the measurement of definite pathophysiological mechanisms across the spectrum of different NDDs. Together with other innovative molecular indicators, NFL will help establish panels of biomarkers-i.e., molecular signaturesencompassing the entire spectrum of molecular events of the NDD spectrum disorders. Applying these molecular signatures in longitudinal investigations will be critical to provide information to depict the pathophysiological processes characterizing different NDDs [198]. These innovative biomarkers will enable the selection of the most appropriate therapies for individual patients by defining which molecular pathophysiological events account for the patient's clinical symptoms at different stages of the disease [199, 200]. This will establish the grounds to develop effective targeted treatment strategies - i.e., "molecularly" targeted therapies - for the accurate treatment of specific molecular pathophysiological pathways. Future developments in investigating NDD heterogeneity will allow clinicians to deliver targeted interventions that are "customized," i.e., tailored, to the definite profiles of the individual NDD patient, according to the precision medicine paradigm. Such a precision medicine-based strategy is now increasingly facing the clinical and biological/genetic complexity and heterogeneity of the various forms of NDD [198]. Precision medicine emphasizes the need of clinical medicine to focus on the pathophysiology of the individual patient, with his/her own distinctive, diverse, and complex matrix of multisystem features [200]. Concerted global efforts will pave the way for a future of neurology, in which drugs will timely and effectively support the prevention and treatment of diseases with very precise biomarker-guided targeted approaches for the right patient at the right time [201].

Acknowledgments H.H. is an employee of Eisai Inc. This work has been performed during his previous position at Sorbonne University, Paris, France. At Sorbonne University, he was supported by the AXA Research Fund, the "Fondation partenariale Sorbonne Université," and the "Fondation pour la Recherche sur Alzheimer," Paris, France.

\section{Compliance with Ethical Standards}

Competing Interests S.L. received lecture honoraria from Roche and Servier.

H.H. is an employee of Eisai Inc. and serves as Senior Associate Editor for the Journal Alzheimer's \& Dementia and does not receive any fees or honoraria since May 2019; before May 2019, he had received lecture fees from Servier, Biogen, and Roche; research grants from Pfizer, Avid, and MSD Avenir (paid to the institution); travel funding from Functional Neuromodulation, Axovant, Eli Lilly and company, Takeda and Zinfandel, GE Healthcare and Oryzon Genomics; and consultancy fees from Qynapse, Jung Diagnostics, Cytox Ltd., Axovant, Anavex, Takeda and Zinfandel, GE Healthcare, Oryzon Genomics, and Functional Neuromodulation, and he also participated in scientific advisory boards of Functional Neuromodulation, Axovant, Eisai, Eli Lilly and company, Cytox Ltd., GE Healthcare, Takeda and Zinfandel, Oryzon Genomics, and Roche Diagnostics.

$\mathrm{He}$ is co-inventor in the following patents as a scientific expert and has received no royalties:

- In Vitro Multiparameter Determination Method for the Diagnosis and Early Diagnosis of Neurodegenerative Disorders Patent Number: 8916388

- In Vitro Procedure for Diagnosis and Early Diagnosis of Neurodegenerative Diseases Patent Number: 8298784

- Neurodegenerative Markers for Psychiatric Conditions Publication Number: 20120196300

- In Vitro Multiparameter Determination Method for the Diagnosis and Early Diagnosis of Neurodegenerative Disorders Publication Number: 20100062463

- In Vitro Method for the Diagnosis and Early Diagnosis of Neurodegenerative Disorders Publication Number: 20100035286

- In Vitro Procedure for Diagnosis and Early Diagnosis of Neurodegenerative Diseases Publication Number: 20090263822

- In Vitro Method for the Diagnosis of Neurodegenerative Diseases Patent Number: 7547553

- CSF Diagnostic in Vitro Method for Diagnosis of Dementias and Neuroinflammatory Diseases Publication Number: 20080206797

- In Vitro Method for the Diagnosis of Neurodegenerative Diseases Publication Number: 20080199966

- Neurodegenerative Markers for Psychiatric Conditions Publication Number: 20080131921
1068

1069

1070

1071

1072

1073

1074

1075

1076

1077

1078

1079

1080

1081

1082

1083

1084

1085

1086

1087

1088

1089

1090

1091

1092

1093

1094

1095

1096

1097

1098

1099

1100

1101

1102

1103

1104

1105

1106 
1107

1108

\section{Q51109 References}

1110

1111

1112

1113

1114

1115

1116

1117

1118

1119

1120

1121

1122

1123

1124

1125

1126

1127

1128

1129

1130

1131

1132

1133

1134

1135

1136

1137

1138

1139

1140

1141

1142

1143

1144

1145

1146

1147

1148

1149

1150

1151

1152

1153

1154

1155

1156

1157

1158

1159

1160

1161

1162

1163

1164

1165

1166

1167
G.P., S.M., A.D.V., G.S., U.B., C.A., R.C., and F.B. declare that they have no conflicts of interest relevant to this work.

1. Glass CK, Saijo K, Winner B, Marchetto MC, Gage FH (2010) Mechanisms underlying inflammation in neurodegeneration. Cell 140(6):918-934

2. Heemels MT (2016) Neurodegenerative diseases. Nature 539(7628): 179

3. Chi H, Chang HY, Sang TK (2018) Neuronal cell death mechanisms in major neurodegenerative diseases. Int J Mol Sci 19(10): 3082

4. Hampel H, Toschi N, Baldacci F et al (2018) Alzheimer's disease biomarker-guided diagnostic workflow using the added value of six combined cerebrospinal fluid candidates: $\mathrm{A} \beta_{1-42}$, total-tau, phosphorylated-tau, NFL, neurogranin, and YKL-40. Alzheimers Dement 14(4):492-501

5. Khalil M, Teunissen CE, Otto M et al (2018) Neurofilaments as biomarkers in neurological disorders. Nat Rev Neurol 14(10): 577-589

6. Gaetani L, Blennow K, Calabresi P, Di Filippo M, Parnetti L, Zetterberg H (2019) Neurofilament light chain as a biomarker in neurological disorders. J Neurol Neurosurg Psychiatry 90(8):870881

7. Zhao Y, Xin Y, Meng S, He Z, Hu W (2019) Neurofilament light chain protein in neurodegenerative dementia: a systematic review and network meta-analysis. Neurosci Biobehav Rev 102:123-138

8. Kanaan NM, Pigino GF, Brady ST, Lazarov O, Binder LI, Morfini GA (2013) Axonal degeneration in Alzheimer's disease: when signaling abnormalities meet the axonal transport system. Exp Neurol 246:44-53

9. Yuan A, Rao MV, Veeranna NRA (2012) Neurofilaments at a glance. J Cell Sci 125:3257-3263

10. Zetterberg H (2016) Neurofilament light: a dynamic cross-disease fluid biomarker for neurodegeneration. Neuron 91(1):1-3

11. Liu Q, Xie F, Siedlak SL et al (2004) Neurofilament proteins in neurodegenerative diseases. Cell Mol Life Sci 61(24):3057-3075

12. Yuan A, Rao MV, Veeranna NRA (2017) Neurofilaments and neurofilament proteins in health and disease. Cold Spring Harb Perspect Biol 9(4):a018309

13. Petzold A, Keir G, Warren J, Fox N, Rossor MN (2007) A systematic review and meta-analysis of CSF neurofilament protein levels as biomarkers in dementia. Neurodegener Dis 4(2-3): 185-194

14. Rosengren LE, Karlsson JE, Karlsson JO, Persson LI, Wikkelsø C (1996) Patients with amyotrophic lateral sclerosis and other neurodegenerative diseases have increased levels of neurofilament protein in CSF. J Neurochem 67(5):2013-2018

15. Landqvist Waldo M, Frizell Santillo A et al (2013) Cerebrospinal fluid neurofilament light chain protein levels in subtypes of frontotemporal dementia. BMC Neurol 13:54

16. Meeter LH, Dopper EG, Jiskoot LC et al (2016) Neurofilament light chain: a biomarker for genetic frontotemporal dementia. Ann Clin Transl Neurol 3(8):623-636

17. Hall S, Öhrfelt A, Constantinescu R et al (2012) Accuracy of a panel of 5 cerebrospinal fluid biomarkers in the differential diagnosis of patients with dementia and/or parkinsonian disorders. Arch Neurol 69(11):1445-1452

18. Magdalinou NK, Paterson RW, Schott JM et al (2015) A panel of nine cerebrospinal fluid biomarkers may identify patients with atypical parkinsonian syndromes. J Neurol Neurosurg Psychiatry 86(11):1240-1247
19. Vinther-Jensen T, Börnsen L, Budtz-Jørgensen E et al (2016) Selected CSF biomarkers indicate no evidence of early neuroinflammation in Huntington disease. Neurol Neuroimmunol Neuroinflamm 3(6):e287

20. Lista S, Toschi N, Baldacci F et al (2017) Diagnostic accuracy of CSF neurofilament light chain protein in the biomarker-guided classification system for Alzheimer's disease. Neurochem Int 108:355-360

21. Zetterberg H, Skillbäck T, Mattsson N et al (2016) Association of cerebrospinal fluid neurofilament light concentration with Alzheimer disease progression. Jama Neurol 73(1):60-67

22. van Eijk JJ, van Everbroeck B, Abdo WF, Kremer BP, Verbeek MM (2010) CSF neurofilament proteins levels are elevated in sporadic Creutzfeldt-Jakob disease. J Alzheimers Dis 21(2):569576

23. Kanata E, Golanska E, Villar-Piqué A et al (2019) Cerebrospinal fluid neurofilament light in suspected sporadic Creutzfeldt-Jakob disease. J Clin Neurosci 60:124-127

24. Lycke JN, Karlsson JE, Andersen O, Rosengren LE (1998) Neurofilament protein in cerebrospinal fluid: a potential marker of activity in multiple sclerosis. J Neurol Neurosurg Psychiatry 64:402-404

25. Yilmaz A, Blennow K, Hagberg L et al (2017) Neurofilament light chain protein as a marker of neuronal injury: review of its use in HIV-1 infection and reference values for HIV-negative controls. Expert Rev Mol Diagn 17(8):761-770

26. Shahim P, Tegner Y, Gustafsson B et al (2016) Neurochemical aftermath of repetitive mild traumatic brain injury. JAMA Neurol 73(11):1308-1315

27. Pujol-Calderón F, Portelius E, Zetterberg H, Blennow K, Rosengren LE, Höglund K (2019) Neurofilament changes in serum and cerebrospinal fluid after acute ischemic stroke. Neurosci Lett 698:58-63

28. Pawlitzki M, Butryn M, Kirchner F et al (2019) CSF Neurofilament light chain level predicts axonal damage in cerebral vasculitis. Ann Clin Transl Neurol 6(6):1134-1137

29. Skillbäck T, Farahmand B, Bartlett JW et al (2014) CSF neurofilament light differs in neurodegenerative diseases and predicts severity and survival. Neurology 83(21):1945-1953

30. Bacioglu M, Maia LF, Preische O et al (2016) Neurofilament light chain in blood and CSF as marker of disease progression in mouse models and in neurodegenerative diseases. Neuron 91:56-66

31. Bridel C, van Wieringen WN, Zetterberg H et al (2019) Diagnostic value of cerebrospinal fluid neurofilament light protein in neurology: a systematic review and meta-analysis. Jama Neurol 76(9):1035-1048

32. Mielke MM, Syrjanen JA, Blennow K et al (2019) Plasma and CSF neurofilament light: relation to longitudinal neuroimaging and cognitive measures. Neurology 93(3):e252-e260

33. Molinuevo JL, Ayton S, Batrla R et al (2018) Current state of Alzheimer's fluid biomarkers. Acta Neuropathol 136(6):821-853

34. Hampel H, Lista S, Khachaturian ZS (2012) Development of biomarkers to chart all Alzheimer's disease stages: the royal road to cutting the therapeutic Gordian Knot. Alzheimers Dement 8(4): 312-336

35. Baldacci F, Lista S, Garaci F, Bonuccelli U, Toschi N, Hampel H (2016) Biomarker-guided classification scheme of neurodegenerative diseases. J Sport Health Sci 5(4):383-387

36. Hampel H, Frank R, Broich K et al (2010) Biomarkers for Alzheimer's disease: academic, industry and regulatory perspectives. Nat Rev Drug Discov 9(7):560-574

37. Blennow K, Hampel H, Weiner M, Zetterberg H (2010) Cerebrospinal fluid and plasma biomarkers in Alzheimer disease. Nat Rev Neurol 6(3):131-144
1168 
1232

1233

1234

1235

1236

1237

1238

1239

1240

1241

1242

1243

1244

1245

1246

1247

1248

1249

1250

1251

1252

1253

1254

1255

1256

1257

1258

1259

1260

1261

1262

1263

1264

1265

1266

1267

1268

1269

1270

1271

1272

1273

1274

1275

1276

1277

1278

1279

1280

1281

1282

1283

1284

1285

1286

1287

1288

1289

1290

1291

1292

1293

1294

1295
38. Zetterberg H, Blennow K (2018) From cerebrospinal fluid to blood: the third wave of fluid biomarkers for Alzheimer's disease. J Alzheimers Dis 64(s1):S271-S279

39. Kuhle J, Barro C, Andreasson U et al (2016) Comparison of three analytical platforms for quantification of the neurofilament light chain in blood samples: ELISA, electrochemiluminescence immunoassay and Simoa. Clin Chem Lab Med 54(10):1655-1661

40. Gaiottino J, Norgren N, Dobson R et al (2013) Increased neurofilament light chain blood levels in neurodegenerative neurological diseases. PLoS One 8(9):e75091

41. Baldacci F, Mazzucchi S, Della Vecchia A et al (2020) The path to biomarker-based diagnostic criteria for the spectrum of neurodegenerative diseases. Expert Rev Mol Diagn 20(4):421-441

42. Califf RM (2018) Biomarker definitions and their applications. Exp Biol Med (Maywood) 243(3):213-221

43. Biomarkers Definitions Working Group (2001) Biomarkers and surrogate endpoints: preferred definitions and conceptual framework. Clin Pharmacol Ther 69(3):89-95

44. Chen XH, Huang S, Kerr D (2011) Biomarkers in clinical medicine. IARC Sci Publ 163:303-322

45. McKhann G, Drachman D, Folstein M, Katzman R, Price D, Stadlan EM (1984) Clinical diagnosis of Alzheimer's disease: report of the NINCDS-ADRDA Work Group under the auspices of Department of Health and Human Services Task Force on Alzheimer's Disease. Neurology 34:939-944

46. McKhann GM, Knopman DS, Chertkow H et al (2011) The diagnosis of dementia due to Alzheimer's disease: recommendations from the National Institute on Aging-Alzheimer's Association workgroups on diagnostic guidelines for Alzheimer's disease. Alzheimers Dement 7:263-269

47. Sperling RA, Aisen PS, Beckett LA et al (2011) Toward defining the preclinical stages of Alzheimer's disease: recommendations from the National Institute on Aging-Alzheimer's Association workgroups on diagnostic guidelines for Alzheimer's disease. Alzheimers Dement 7:280-292

48. Albert MS, DeKosky ST, Dickson D et al (2011) The diagnosis of mild cognitive impairment due to Alzheimer's disease: recommendations from the National Institute on Aging-Alzheimer's Association workgroups on diagnostic guidelines for Alzheimer's disease. Alzheimers Dement 7:270-279

49. Dubois B, Feldman HH, Jacova C et al (2014) Advancing research diagnostic criteria for Alzheimer's disease: the IWG-2 criteria. Lancet Neurol 13:614-629

50. Brooks BR (1994) El Escorial World Federation of Neurology criteria for the diagnosis of amyotrophic lateral sclerosis. Subcommittee on Motor Neuron Diseases/Amyotrophic Lateral Sclerosis of the World Federation of Neurology Research Group on Neuromuscular Diseases and the El Escorial "Clinical limits of amyotrophic lateral sclerosis" workshop contributors. J Neurol Sci 124(Suppl):96-107

51. Costa J, Swash M, de Carvalho M (2012) Awaji criteria for the diagnosis of amyotrophic lateral sclerosis: a systematic review. Arch Neurol 69:1410-1416

52. Al-Chalabi A, Hardiman O, Kiernan MC, Chiò A, Rix-Brooks B, van den Berg LH (2016) Amyotrophic lateral sclerosis: moving towards a new classification system. Lancet Neurol 15(11):11821194

53. McKeith IG, Galasko D, Kosaka K et al (1996) Consensus guidelines for the clinical and pathologic diagnosis of dementia with Lewy bodies (DLB): report of the consortium on DLB international workshop. Neurology 47(5):1113-1124

54. McKeith IG, Dickson DW, Lowe J et al (2005) Diagnosis and management of dementia with Lewy bodies: third report of the DLB Consortium. Neurology 65(12):1863-1872
55. McKeith IG, Boeve BF, Dickson DW et al (2017) Diagnosis and management of dementia with Lewy bodies: fourth consensus report of the DLB Consortium. Neurology 89(1):88-100

56. Neary D, Snowden JS, Gustafson L et al (1998) Frontotemporal lobar degeneration: a consensus on clinical diagnostic criteria. Neurology 51:1546-1554

57. Piguet O, Hornberger M, Mioshi E, Hodges JR (2011) Behavioural-variant frontotemporal dementia: diagnosis, clinical staging, and management. Lancet Neurol 10:162-172

58. Gorno-Tempini ML, Hillis AE, Weintraub S et al (2011) Classification of primary progressive aphasia and its variants. Neurology 76:1006-1014

59. Gibb WR, Lees AJ (1988) The relevance of the Lewy body to the pathogenesis of idiopathic Parkinson's disease. J Neurol Neurosurg Psychiatry 51:745-752

60. Gelb DJ, Oliver E, Gilman S (1999) Diagnostic criteria for Parkinson disease. Arch Neurol 56(1):33-39

61. Postuma RB, Berg D, Stern M et al (2015) MDS clinical diagnostic criteria for Parkinson's disease: MDS-PD clinical diagnostic criteria. Mov Disord 30(12):1591-1601

62. Litvan I, Agid Y, Calne D et al (1996) Clinical research criteria for the diagnosis of progressive supranuclear palsy (SteeleRichardson-Olszewski syndrome): report of the NINDS-SPSP international workshop. Neurology 47:1-9

63. Gilman S, Wenning GK, Low PA et al (2008) Second consensus statement on the diagnosis of multiple system atrophy. Neurology 71(9):670-676

64. Armstrong MJ, Litvan I, Lang AE et al (2013) Criteria for the diagnosis of corticobasal degeneration. Neurology 80(5):496-503

65. Hoglinger GU, Respondek G, Stamelou M et al (2017) Clinical diagnosis of progressive supranuclear palsy: the movement disorder society criteria. Mov Disord 32:853-864

66. World Health Organization (2003) WHO manual for surveillance of human transmissible spongiform encephalopathies, including variant Creutzfeldt-Jakob disease https://apps.who.int/iris/handle/ 10665/42656 [Accessed 30 Jan 2003]

67. Manix M, Kalakoti P, Henry M et al (2015) Creutzfeldt-Jakob disease: updated diagnostic criteria, treatment algorithm, and the utility of brain biopsy. Neurosurg Focus 39:E2

68. Xia J, Broadhurst DI, Wilson M, Wishart DS (2013) Translational biomarker discovery in clinical metabolomics: an introductory tutorial. Metabolomics 9(2):280-299

69. Winblad B, Amouyel P, Andrieu S et al (2016) Defeating Alzheimer's disease and other dementias: a priority for European science and society. Lancet Neurol 15(5):455-532

70. Fogel DB (2018) Factors associated with clinical trials that fail and opportunities for improving the likelihood of success: a review. Contemp Clin Trials Commun 11:156-164

71. Dubois B (2018) The emergence of a new conceptual framework for Alzheimer's disease. J Alzheimers Dis 62(3):1059-1066

72. Beach TG, Monsell SE, Phillips LE, Kukull W (2012) Accuracy of the clinical diagnosis of Alzheimer disease at National Institute on Aging Alzheimer Disease Centers, 2005-2010. J Neuropathol Exp Neurol 71(4):266-273

73. Jack CR Jr, Bennett DA, Blennow K et al (2016) A/T/N: an unbiased descriptive classification scheme for Alzheimer disease biomarkers. Neurology 87(5):539-547

74. Jack CR Jr, Bennett DA, Blennow K et al (2018) NIA-AA research framework: toward a biological definition of Alzheimer's disease. Alzheimers Dement 14(4):535-562

75. Hampel H, O’Bryant SE, Molinuevo JL et al (2018) Blood-based biomarkers for Alzheimer disease: mapping the road to the clinic. Nat Rev Neurol 14(11):639-652

76. Ashton NJ, Leuzy A, Lim YM et al (2019) Increased plasma neurofilament light chain concentration correlates with severity 
1361

1362

1363

1364

1365

1366

1367

1368

1369

1370

1371

1372

1373

1374

1375

1376

1377

1378

1379

1380

1381

1382

1383

1384

1385

1386

1387

1388

1389

1390

1391

1392

1393

1394

1395

1396

1397

1398

1399

1400

1401

1402

1403

1404

1405

1406

1407

1408

1409

1410

1411

1412

1413

1414

1415

1416

1417

1418

1419

1420

1421

Q6 1422

1423

1424

1425 of post-mortem neurofibrillary tangle pathology and neurodegeneration. Acta Neuropathol Commun 7(1):5

77. Mattsson N, Andreasson U, Zetterberg H, Blennow K, Alzheimer's Disease Neuroimaging Initiative (2017) Association of plasma neurofilament light with neurodegeneration in patients with Alzheimer disease. Jama Neurol 74(5):557566

78. Vågberg M, Norgren N, Dring A et al (2015) Levels and age dependency of neurofilament light and glial fibrillary acidic protein in healthy individuals and their relation to the brain parenchymal fraction. PLoS One 10(8):e0135886

79. Khalil M, Pirpamer L, Hofer E et al (2020) Serum neurofilament light levels in normal aging and their association with morphologic brain changes. Nat Commun 11(1):812

80. Forgrave LM, Ma M, Best JR, DeMarco ML (2019) The diagnostic performance of neurofilament light chain in CSF and blood for Alzheimer's disease, frontotemporal dementia, and amyotrophic lateral sclerosis: a systematic review and meta-analysis. Alzheimers Dement (Amst) 11:730-743

81. Lewczuk P, Ermann N, Andreasson U et al (2018) Plasma neurofilament light as a potential biomarker of neurodegeneration in Alzheimer's disease. Alzheimers Res Ther 10(1):71

82. Mattsson N, Cullen NC, Andreasson U, Zetterberg H, Blennow K (2019) Association between longitudinal plasma neurofilament light and neurodegeneration in patients with Alzheimer disease. JAMA Neurol 76(7):791-799

83. Pereira JB, Westman E, Hansson O, Alzheimer's Disease Neuroimaging Initiative (2017) Association between cerebrospinal fluid and plasma neurodegeneration biomarkers with brain atrophy in Alzheimer's disease. Neurobiol Aging 58:14-29

84. Lin YS, Lee WJ, Wang SJ, Fuh JL (2018) Levels of plasma neurofilament light chain and cognitive function in patients with Alzheimer or Parkinson disease. Sci Rep 8(1): 17368

85. Wang SY, Chen W, Xu W et al (2019) Neurofilament light chain in cerebrospinal fluid and blood as a biomarker for neurodegenerative diseases: a systematic review and meta-analysis. J Alzheimers Dis 72(4):1353-1361

86. Zhou W, Zhang J, Ye F et al (2017) Plasma neurofilament light chain levels in Alzheimer's disease. Neurosci Lett 650:60-64

87. Benedet AL, Ashton NJ, Pascoal TA et al (2019) Plasma neurofilament light associates with Alzheimer's disease metabolic decline in amyloid-positive individuals. Alzheimers Dement (Amst) 11:679-689

88. Timmers M, Tesseur I, Bogert J et al (2019) Relevance of the interplay between amyloid and tau for cognitive impairment in early Alzheimer's disease. Neurobiol Aging 79:131-141

89. Preische O, Schultz SA, Apel A et al (2019) Serum neurofilament dynamics predicts neurodegeneration and clinical progression in presymptomatic Alzheimer's disease. Nat Med 25(2):277-283

90. $\mathrm{Hu} \mathrm{H}, \mathrm{Chen} \mathrm{KL}, \mathrm{Ou} \mathrm{YN}$ et al (2019) Neurofilament light chain plasma concentration predicts neurodegeneration and clinical progression in nondemented elderly adults. Aging (Albany NY) 11(17):6904-6914

91. Chatterjee P, Goozee K, Sohrabi HR et al (2018) Association of plasma neurofilament light chain with neocortical amyloid- $\beta$ load and cognitive performance in cognitively normal elderly participants. J Alzheimers Dis 63(2):479-487

92. Mayeli M, Mirshahvalad SM, Aghamollaii V, Tafakhori A, Abdolalizadeh A, Rahmani F (2019) Plasma neurofilament light chain levels are associated with cortical hypometabolism in Alzheimer disease signature regions. J Neuropathol Exp Neurol: nlz054

93. Weston PSJ, Poole T, Ryan NS et al (2017) Serum neurofilament light in familial Alzheimer disease: a marker of early neurodegeneration. Neurology 89(21):2167-2175
94. Rascovsky K, Hodges JR, Knopman D et al (2011) Sensitivity of revised diagnostic criteria for the behavioural variant of frontotemporal dementia. Brain 134:2456-2477

95. Erkkinen MG, Kim MO, Geschwind MD (2018) Clinical neurology and epidemiology of the major neurodegenerative diseases. Cold Spring Harb Perspect Biol 10(4):a033118

96. Bang J, Spina S, Miller BL (2015) Frontotemporal dementia. Lancet 386:1672-1682

97. Spinelli EG, Mandelli ML, Miller ZA et al (2017) Typical and atypical pathology in primary progressive aphasia variants. Ann Neurol 81:430-443

98. Strong MJ, Abrahams S, Goldstein LH et al (2017) Amyotrophic lateral sclerosis - frontotemporal spectrum disorder (ALS-FTSD): revised diagnostic criteria. Amyotroph Lateral Scler Frontotemporal Degener 18:153-174

99. Rademakers R, Neumann M, Mackenzie IR (2012) Advances in understanding the molecular basis of frontotemporal dementia. Nat Rev Neurol 8(8):423-434

100. Ducharme S, Dols A, Laforce R et al (2020) Recommendations to distinguish behavioural variant frontotemporal dementia from psychiatric disorders. Brain 143(6):1632-1650

101. Meeter LH, Kaat LD, Rohrer JD, van Swieten JC (2017) Imaging and fluid biomarkers in frontotemporal dementia. Nat Rev Neurol 13(7):406-419

102. Zetterberg H, van Swieten JC, Boxer AL, Rohrer JD (2019) Review: Fluid biomarkers for frontotemporal dementias. Neuropathol Appl Neurobiol 45(1):81-87

103. Pijnenburg YA, Verwey NA, van der Flier WM, Scheltens P, Teunissen CE (2015) Discriminative and prognostic potential of cerebrospinal fluid phosphoTau/tau ratio and neurofilaments for frontotemporal dementia subtypes. Alzheimers Dement (Amst) 1(4):505-512

104. Ende E, Meeter L, Poos J et al (2019) Serum neurofilament light chain in genetic frontotemporal dementia: a longitudinal, multicentre cohort study. Lancet Neurol 18:1103-1111

105. Steinacker P, Semler E, Anderl-Straub S et al (2017) Neurofilament as a blood marker for diagnosis and monitoring of primary progressive aphasias. Neurology 88:961-969

106. Al Shweiki MR, Steinacker P, Oeckl P et al (2019) Neurofilament light chain as a blood biomarker to differentiate psychiatric disorders from behavioural variant frontotemporal dementia. J Psychiatr Res 113:137-140

107. Katisko K, Cajanus A, Jääskeläinen O et al (2020) Serum neurofilament light chain is a discriminative biomarker between frontotemporal lobar degeneration and primary psychiatric disorders. J Neurol 267(1):162-167

108. Wilke C, Preische O, Deuschle C et al (2016) Neurofilament light chain in FTD is elevated not only in cerebrospinal fluid, but also in serum. J Neurol Neurosurg Psychiatry 87:1270-1272

109. Steinacker P, Anderl-Straub S, Diehl-Schmid J et al (2018) Serum neurofilament light chain in behavioral variant frontotemporal dementia. Neurology 91(15):e1390-e1401

110. Matias-Guiu JA, Gomez-Pinedo U, Forero L et al (2019) Plasma neurofilament light chain in primary progressive aphasia and related disorders: clinical significance and metabolic correlates. J Alzheimers Dis 72:1-10

111. Cellura E, Spataro R, Taiello AC, La Bella V (2012) Factors affecting the diagnostic delay in amyotrophic lateral sclerosis. Clin Neurol Neurosurg 114(6):550-554

112. Turner MR, Kiernan MC, Leigh PN, Talbot K (2009) Biomarkers in amyotrophic lateral sclerosis. Lancet Neurol 8(1):94-109

113. Lehnert S, Costa J, de Carvalho M et al (2014) Multicentre quality control evaluation of different biomarker candidates for amyotrophic lateral sclerosis. Amyotroph Lateral Scler Frontotemporal Degener 15(5-6):344-350 
1491

1492

1493

1494

1495

1496

1497

1498

1499

1500

1501

1502

1503

1504

1505

1506

1507

1508

1509

1510

1511

1512

1513

1514

1515

1516

1517

1518

1519

1520

1521

1522

1523

1524

1525

1526

1527

1528

1529

1530

1531

1532

1533

1534

1535

1536

1537

1538

1539

1540

1541

1542

1543

1544

1545

1546

1547

1548

1549

1550

1551

1552

1553

1554

1555
114. Turner MR, Benatar M (2015) Ensuring continued progress in biomarkers for amyotrophic lateral sclerosis. Muscle Nerve 51(1):14-18

115. Xu Z, Henderson RD, David M, McCombe PA (2016) Neurofilaments as biomarkers for amyotrophic lateral sclerosis: a systematic review and meta-analysis. PLoS One 11(10): e0164625

116. Turner MR, Gray E (2016) Are neurofilaments heading for the ALS clinic? J Neurol Neurosurg Psychiatry 87(1):3-4

117. Brettschneider J, Petzold A, Süssmuth SD, Ludolph AC, Tumani $H$ (2006) Axonal damage markers in cerebrospinal fluid are increased in ALS. Neurology 66(6):852-856

118. Lin H, Schlaepfer WW (2006) Role of neurofilament aggregation in motor neuron disease. Ann Neurol 60(4):399-406

119. Munoz DG, Greene C, Perl DP, Selkoe DJ (1988) Accumulation of phosphorylated neurofilaments in anterior horn motoneurons of amyotrophic lateral sclerosis patients. J Neuropathol Exp Neurol 47(1):9-18

120. Collard JF, Côté F, Julien JP (1995) Defective axonal transport in a transgenic mouse model of amyotrophic lateral sclerosis. Nature 375(6526):61-64

121. Lee MK, Marszalek JR, Cleveland DW (1994) A mutant neurofilament subunit causes massive, selective motor neuron death: implications for the pathogenesis of human motor neuron disease. Neuron 13(4):975-988

122. Cañete-Soler R, Silberg DG, Gershon MD, Schlaepfer WW (1999) Mutation in neurofilament transgene implicates RNA processing in the pathogenesis of neurodegenerative disease. $\mathrm{J}$ Neurosci 19(4):1273-1283

123. Nie Z, Wu J, Zhai J et al (2002) Untranslated element in neurofilament mRNA has neuropathic effect on motor neurons of transgenic mice. J Neurosci 22(17):7662-7670

124. Zhai J, Lin H, Julien JP, Schlaepfer WW (2007) Disruption of neurofilament network with aggregation of light neurofilament protein: a common pathway leading to motor neuron degeneration due to Charcot-Marie-Tooth disease-linked mutations in NFL and HSPB1. Hum Mol Genet 16(24):3103-3116

125. Lin H, Zhai J, Schlaepfer WW (2005) RNA-binding protein is involved in aggregation of light neurofilament protein and is implicated in the pathogenesis of motor neuron degeneration. Hum Mol Genet 14(23):3643-3659

126. Watson D (1991) Regional variation in the abundance of axonal cytoskeletal proteins. J Neurosci Res 30(1):226-231

127. Reijn TS, Abdo WF, Schelhaas HJ, Verbeek MM (2009) CSF neurofilament protein analysis in the differential diagnosis of ALS. J Neurol 256(4):615-619

128. Steinacker P, Feneberg E, Weishaupt J et al (2016) Neurofilaments in the diagnosis of motoneuron diseases: a prospective study on 455 patients. J Neurol Neurosurg Psychiatry 87(1):12-20

129. Tortelli R, Ruggieri M, Cortese R et al (2012) Elevated cerebrospinal fluid neurofilament light levels in patients with amyotrophic lateral sclerosis: a possible marker of disease severity and progression. Eur J Neurol 19(12):1561-1567

130. Zetterberg H, Jacobsson J, Rosengren L, Blennow K, Andersen PM (2007) Cerebrospinal fluid neurofilament light levels in amyotrophic lateral sclerosis: impact of SOD1 genotype. Eur J Neurol 14(12):1329-1333

131. Tortelli R, Copetti M, Ruggieri M et al (2015) Cerebrospinal fluid neurofilament light chain levels: marker of progression to generalized amyotrophic lateral sclerosis. Eur J Neurol 22(1):215-218

132. Poesen K, Van Damme P (2019) Diagnostic and prognostic performance of neurofilaments in ALS. Front Neurol 9:1167

133. Menke RA, Gray E, Lu CH et al (2015) CSF neurofilament light chain reflects corticospinal tract degeneration in ALS. Ann Clin Transl Neurol 2(7):748-755
134. Feneberg E, Oeckl P, Steinacker P et al (2018) Multicenter evaluation of neurofilaments in early symptom onset amyotrophic lateral sclerosis. Neurology 90(1):e22-e30

135. Gille B, De Schaepdryver M, Goossens J et al (2019) Serum neurofilament light chain levels as a marker of upper motor neuron degeneration in patients with amyotrophic lateral sclerosis. Neuropathol Appl Neurobiol 45(3):291-304

136. Lu CH, Macdonald-Wallis C, Gray E et al (2015) Neurofilament light chain: a prognostic biomarker in amyotrophic lateral sclerosis. Neurology 84(22):2247-2257

137. Gaiani A, Martinelli I, Bello L et al (2017) Diagnostic and prognostic biomarkers in amyotrophic lateral sclerosis: neurofilament light chain levels in definite subtypes of disease. Jama Neurol 74(5):525-532

138. Steinacker P, Huss A, Mayer B et al (2017) Diagnostic and prognostic significance of neurofilament light chain NF-L, but not progranulin and $\mathrm{S} 100 \mathrm{~B}$, in the course of amyotrophic lateral sclerosis: data from the German MND-net. Amyotroph Lateral Scler Frontotemporal Degener 18(1-2):112-119

139. Verde F, Steinacker P, Weishaupt JH et al (2019) Neurofilament light chain in serum for the diagnosis of amyotrophic lateral sclerosis. J Neurol Neurosurg Psychiatry 90(2):157-164

140. Thouvenot E, Demattei C, Lehmann S et al (2020) Serum neurofilament light chain at time of diagnosis is an independent prognostic factor of survival in amyotrophic lateral sclerosis. Eur J Neurol 27(2):251-257

141. Poesen K, De Schaepdryver M, Stubendorff B et al (2017) Neurofilament markers for ALS correlate with extent of upper and lower motor neuron disease. Neurology 88(24):2302-2309

142. De Schaepdryver M, Lunetta C, Tarlarini C et al (2020) Neurofilament light chain and $\mathrm{C}$ reactive protein explored as predictors of survival in amyotrophic lateral sclerosis. J Neurol Neurosurg Psychiatry 91(4):436-437

143. Weydt P, Oeckl P, Huss A et al (2016) Neurofilament levels as biomarkers in asymptomatic and symptomatic familial amyotrophic lateral sclerosis. Ann Neurol 79(1):152-158

144. Benatar M, Wuu J, Andersen PM, Lombardi V, Malaspina A (2018) Neurofilament light: a candidate biomarker of presymptomatic amyotrophic lateral sclerosis and phenoconversion. Ann Neurol 84(1):130-139

145. Olsson B, Alberg L, Cullen NC et al (2019) NFL is a marker of treatment response in children with SMA treated with nusinersen. J Neurol 266(9):2129-2136

146. Corey DR (2017) Nusinersen, an antisense oligonucleotide drug for spinal muscular atrophy. Nat Neurosci 20(4):497-499

147. Wurster CD, Günther R, Steinacker P et al (2019) Neurochemical markers in CSF of adolescent and adult SMA patients undergoing nusinersen treatment. Ther Adv Neurol Disord 12: 1756286419846058

148. Fratta P, Nirmalananthan N, Masset L et al (2014) Correlation of clinical and molecular features in spinal bulbar muscular atrophy. Neurology 82(23):2077-2084

149. Respondek G, Roeber S, Kretzschmar H et al (2013) Accuracy of the National Institute for Neurological Disorders and Stroke/Society for Progressive Supranuclear Palsy and neuroprotection and natural history in Parkinson plus syndromes criteria for the diagnosis of progressive supranuclear palsy. Mov Disord 28:504-509

150. Rizzo G, Copetti M, Arcuti S, Martino D, Fontana A, Logroscino G (2016) Accuracy of clinical diagnosis of Parkinson disease: a systematic review and meta-analysis. Neurology 86(6):566-576

151. Rizzo G, Arcuti S, Copetti M et al (2018) Accuracy of clinical diagnosis of dementia with Lewy bodies: a systematic review and meta-analysis. J Neurol Neurosurg Psychiatry 89(4):358-366

152. Miki Y, Foti SC, Asi YT et al (2019) Improving diagnostic accuracy of multiple system atrophy: a clinicopathological study. Brain 142(9):2813-2827
1556 
1622

1623

1624

1625

1626

1627

1628

1629

1630

1631

1632

1633

1634

1635

1636

1637

1638

1639

1640

1641

1642

1643

1644

1645

1646

1647

1648

1649

1650

1651

1652

1653

1654

1655

1656

1657

1658

1659

1660

1661

1662

1663

1664

1665

1666

1667

1668

1669

1670

1671

1672

1673

1674

1675

1676

1677

1678

1679

1680

1681

1682

1683

1684

1685

1686

1687
153. Abdo WF, Bloem BR, Van Geel WJ, Esselink RA, Verbeek MM (2007) CSF neurofilament light chain and tau differentiate multiple system atrophy from Parkinson's disease. Neurobiol Aging 28(5):742-747

154. Herbert MK, Aerts MB, Beenes M et al (2015) CSF neurofilament light chain but not FLT3 ligand discriminates parkinsonian disorders. Front Neurol 6:91

155. Tsukamoto K, Matsusue E, Kanasaki Y et al (2012) Significance of apparent diffusion coefficient measurement for the differential diagnosis of multiple system atrophy, progressive supranuclear palsy, and Parkinson's disease: evaluation by 3.0-T MR imaging. Neuroradiology 54:947-955

156. Hansson O, Janelidze S, Hall S et al (2017) Blood-based NfL: a biomarker for differential diagnosis of parkinsonian disorder. Neurology 88:930-937

157. Parnetti L, Gaetani L, Eusebi P et al (2019) CSF and blood biomarkers for Parkinson's disease. Lancet Neurol 18(6):573-586

158. Marques TM, van Rumund A, Oeckl P et al (2019) Serum NFL discriminates Parkinson disease from atypical parkinsonisms. Neurology 92(13):e1479-e1486

159. Oosterveld LP, Verberk IMW, Majbour NK et al (2020) CSF or serum neurofilament light added to $\alpha$-synuclein panel discriminates Parkinson's from controls. Mov Disord 35(2):288-295

160. Ng ASL, Tan YJ, Yong ACW et al (2020) Utility of plasma neurofilament light as a diagnostic and prognostic biomarker of the postural instability gait disorder motor subtype in early Parkinson's disease. Mol Neurodegener 15(1):33

161. Mollenhauer B, Dakna M, Liu T-Y et al (2019) Validation of serum neurofilament light chain as a biomarker of Parkinson's disease progression. bioRxiv. Cold Spring Harbor Laboratory; Epub:762237. https://doi.org/10.1101/762237

162. Lin CH, Li CH, Yang KC et al (2019) Blood NfL: a biomarker for disease severity and progression in Parkinson disease. Neurology 93(11):e1104-e1111

163. Rojas JC, Karydas A, Bang J et al (2016) Plasma neurofilament light chain predicts progression in progressive supranuclear palsy. Ann Clin Transl Neurol 3:216-225

164. Donker Kaat L, Meeter LH, Chiu WZ et al (2018) Serum neurofilament light chain in progressive supranuclear palsy. Parkinsonism Relat Disord 56:98-101

165. Wilke C, Bender F, Hayer SN et al (2018) Serum neurofilament light is increased in multiple system atrophy of cerebellar type and in repeat-expansion spinocerebellar ataxias: a pilot study. J Neurol 265(7):1618-1624

166. Abdo WF, van de Warrenburg BP, Munneke M et al (2006) CSF analysis differentiates multiple-system atrophy from idiopathic late-onset cerebellar ataxia. Neurology 67:474-479

167. Li QF, Dong Y, Yang L et al (2019) Neurofilament light chain is a promising serum biomarker in spinocerebellar ataxia type 3. Mol Neurodegener 14(1):39

168. Zeitlberger AM, Thomas-Black G, Garcia-Moreno H et al (2018) Plasma markers of neurodegeneration are raised in Friedreich's ataxia. Front Cell Neurosci 12:366

169. Hayer SN, Liepelt I, Barro C et al (2020) NfL and pNfH are increased in Friedreich's ataxia. J Neurol 267(5):1420-1430

170. Johnson CD, Davidson BL (2010) Huntington's disease: progress toward effective disease-modifying treatments and a cure. Hum Mol Genet 19(R1):R98-R102

171. Bates GP, Dorsey R, Gusella JF et al (2015) Huntington disease. Nat Rev Dis Primers 1:15005

172. Craufurd D, MacLeod R, Frontali M et al (2015) Diagnostic genetic testing for Huntington's disease. Pract Neurol 15(1):80-84

173. Constantinescu R, Romer M, Oakes D, Rosengren L, Kieburtz K (2009) Levels of the light subunit of neurofilament triplet protein in cerebrospinal fluid in Huntington's disease. Parkinsonism Relat Disord 15(3):245-248
174. Niemelä V, Landtblom AM, Blennow K, Sundblom J (2017) Tau or neurofilament light-which is the more suitable biomarker for Huntington's disease? PLoS One 12(2):e0172762

175. Ross CA, Aylward EH, Wild EJ et al (2014) Huntington disease: natural history, biomarkers and prospects for therapeutics. Nat Rev Neurol 10(4):204-216

176. Wild EJ, Boggio R, Langbehn D et al (2015) Quantification of mutant huntingtin protein in cerebrospinal fluid from Huntington's disease patients. J Clin Invest 125(5):1979-1986

177. Byrne LM, Rodrigues FB, Blennow K et al (2017) Neurofilament light protein in blood as a potential biomarker of neurodegeneration in Huntington's disease: a retrospective cohort analysis. Lancet Neurol 16(8):601-609

178. Rodrigues FB, Byrne LM, Wild EJ (2018) Biofluid biomarkers in Huntington's disease. Methods Mol Biol 1780:329-396

179. Johnson EB, Byrne LM, Gregory S et al (2018) Neurofilament light protein in blood predicts regional atrophy in Huntington disease. Neurology 90(8):e717-e723

180. Byrne LM, Rodrigues FB, Johnson EB et al (2018) Evaluation of mutant huntingtin and neurofilament proteins as potential markers in Huntington's disease. Sci Transl Med 10(458):eaat7108

181. Uttley L, Carroll C, Wong R, Hilton DA, Stevenson M (2020) Creutzfeldt-Jakob disease: a systematic review of global incidence, prevalence, infectivity, and incubation. Lancet Infect Dis 20(1):e2-e10

182. Parchi P, Giese A, Capellari S et al (1999) Classification of sporadic Creutzfeldt-Jakob disease based on molecular and phenotypic analysis of 300 subjects. Ann Neurol 46(2):224-233

183. Zerr I, Kallenberg K, Summers DM et al (2009) Updated clinical diagnostic criteria for sporadic Creutzfeldt-Jakob disease. Brain 132:2659-2668

184. Zanusso G, Fiorini M, Ferrari S et al (2011) Cerebrospinal fluid markers in sporadic Creutzfeldt-Jakob disease. Int J Mol Sci 12: 6281-6292

185. Steinacker P, Blennow K, Halbgebauer S et al (2016) Neurofilaments in blood and CSF for diagnosis and prediction of onset in Creutzfeldt-Jakob disease. Sci Rep 6:38737

186. Zerr I, Schmitz M, Karch A et al (2018) Cerebrospinal fluid neurofilament light levels in neurodegenerative dementia: evaluation of diagnostic accuracy in the differential diagnosis of prion diseases. Alzheimers Dement 14(6):751-763

187. Abu-Rumeileh S, Capellari S, Stanzani-Maserati M et al (2018) The CSF neurofilament light signature in rapidly progressive neurodegenerative dementias. Alzheimers Res Ther 10(1):3

188. Thompson AGB, Luk C, Heslegrave AJ et al (2018) Neurofilament light chain and tau concentrations are markedly increased in the serum of patients with sporadic CreutzfeldtJakob disease, and tau correlates with rate of disease progression. J Neurol Neurosurg Psychiatry 89(9):955-961

189. Kovacs GG, Andreasson U, Liman V et al (2017) Plasma and cerebrospinal fluid tau and neurofilament concentrations in rapidly progressive neurological syndromes: a neuropathology-based cohort. Eur J Neurol 24(11):1326-1e77

190. Staffaroni AM, Kramer AO, Casey M et al (2019) Association of blood and cerebrospinal fluid tau level and other biomarkers with survival time in sporadic Creutzfeldt-Jakob disease. JAMA Neurol 76(8):969-977

191. Ladogana A, Kovacs GG (2018) Genetic Creutzfeldt-Jakob disease. Handb Clin Neurol 153:219-242

192. Kim MO, Takada LT, Wong K, Forner SA, Geschwind MD (2018) Genetic PrP prion diseases. Cold Spring Harb Perspect Biol 10(5):a033134

193. Vallabh SM, Minikel EV, Schreiber SL, Lander ES (2020) Towards a treatment for genetic prion disease: trials and biomarkers. Lancet Neurol 19(4):361-368
1688 
1753

1754

1755

1756

1757

1758

1759

1760

1761

1762

1763

1764

1765

1766

1767

1768

1780
194. Baldacci F, Lista S, Vergallo A, Palermo G, Giorgi FS, Hampel H (2019) A frontline defense against neurodegenerative diseases: the development of early disease detection methods. Expert Rev Mol Diagn 19(7):559-563

195. Ashton NJ, Hye A, Rajkumar AP et al (2020) An update on bloodbased biomarkers for non-Alzheimer neurodegenerative disorders. Nat Rev Neurol 16(5):265-284

196. Hampel H, Lista S (2013) Use of biomarkers and imaging to assess pathophysiology, mechanisms of action and target engagement. J Nutr Health Aging 17(1):54-63

197. Hampel H, O'Bryant SE, Castrillo JI et al (2016) Precision medicine - the golden gate for detection, treatment and prevention of Alzheimer's disease. J Prev Alzheimers Dis 3(4):243-259

198. Hampel H, Toschi N, Babiloni C et al (2018) Revolution of Alzheimer precision neurology. Passageway of systems biology and neurophysiology. J Alzheimers Dis 64(s1):S47-S105
199. Hampel H, O’Bryant SE, Durrleman S et al (2017) A precision medicine initiative for Alzheimer's disease: the road ahead to biomarker-guided integrative disease modeling. Climacteric 20(2):107-118

200. Hampel H, Vergallo A, Aguilar LF et al (2018) Precision pharmacology for Alzheimer's disease. Pharmacol Res 130:331-365

201. Hampel H, Vergallo A, Perry G, Lista S, Alzheimer Precision Medicine Initiative (APMI) (2019) The Alzheimer Precision Medicine Initiative. J Alzheimers Dis 68(1):1-24

Publisher's Note Springer Nature remains neutral with regard to jurisdictional claims in published maps and institutional affiliations.
1769

1770

1771

1772

1773

1774

1775

1776

1777

1778 


\section{AUTHOR QUERIES}

AUTHOR PLEASE ANSWER ALL QUERIES.

Q1. Please check if the affiliations are captured and presented correctly.

Q2. Please check if the section headings are assigned to appropriate levels.

Q3. Please check the provided expanded form for the abbreviation "MMSE" if correct.

Q4. Please check the word "diseases" inserted after the word "neurodegenerative," which can be found in the sentence starting "Finally, serum NFL levels were reported relatively lower," for completeness if correct.

Q5. References [19, 174], [22, 186], [200, 203] based on original manuscript we received were identical. Hence, the latter was deleted and reference list and citations were adjusted. Please check if appropriate.

Q6. Please provide complete bibliographic details of this reference. 